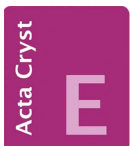

CRYSTALLOGRAPHIC COMMUNICATIONS

ISSN 2056-9890

Received 20 January 2020

Accepted 28 February 2020

Edited by P. Roussel, ENSCL, France

Keywords: crystal structure; rare-earth silicide; chiral structure; chiral magnet; single-crystal growth; X-ray diffraction.

CCDC references: 1974014; 1974013

Supporting information: this article has supporting information at journals.iucr.org/e

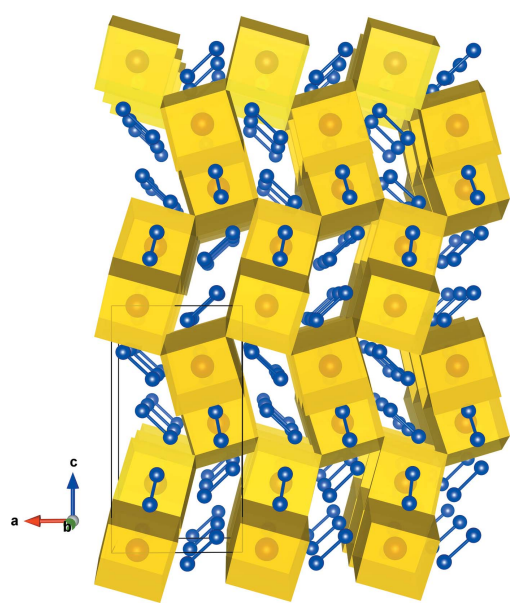

OPEN ๑ ACCESS

\section{Redetermination of the crystal structure of $R_{5} \mathrm{Si}_{4}(R=\mathrm{Pr}, \mathrm{Nd})$ from single-crystal X-ray diffraction data}

\author{
Kaori Yokota, ${ }^{a}$ Ryuta Watanuki, ${ }^{\text {b* }}$ Miki Nakashima, ${ }^{a}$ Masatomo Uehara, ${ }^{a}$ Jun \\ Gouchi, ${ }^{c}$ Yoshiya Uwatoko ${ }^{c}$ and Izuru Umehara ${ }^{a}$
}

\begin{abstract}
a Department of Physics, Yokohama National University, 79-5 Tokiwadai, Hodogaya-ku, Yokohama 240-8501, Japan, b Department of Chemistry, Yokohama National University, 79-5 Tokiwadai, Hodogaya-ku, Yokohama 240-8501, Japan, and ${ }^{\mathbf{C}}$ The Institute for Solid State Physics, The University of Tokyo, 5-1-5, Kashiwanoha, Kashiwa, Chiba 277-8581, Japan. *Correspondence e-mail: watanuki-ryuta-sm@ynu.ac.jp
\end{abstract}

The crystal structures of praseodymium silicide (5/4), $\operatorname{Pr}_{5} \mathrm{Si}_{4}$, and neodymium silicide (5/4), $\mathrm{Nd}_{5} \mathrm{Si}_{4}$, were redetermined using high-quality single-crystal X-ray diffraction data. The previous structure reports of $\operatorname{Pr}_{5} \mathrm{Si}_{4}$ were only based on powder X-ray diffraction data [Smith et al. (1967). Acta Cryst. 22 940-943; Yang et al. (2002b). J. Alloys Compd. 339, 189-194; Yang et al., (2003). J. Alloys Compd. 263, 146-153]. On the other hand, the structure of $\mathrm{Nd}_{5} \mathrm{Si}_{4}$ has been determined from powder data [neutron; Cadogan et al., (2002). J. Phys. Condens. Matter, 14, 7191-7200] and X-ray [Smith et al. (1967). Acta Cryst. 22 940-943; Yang et al. (2002b). J. Alloys Compd. 339, 189-194; Yang et al., (2003). J. Alloys Compd. 263, 146-153] and single-crystal data with isotropic atomic displacement parameters [Roger et al., (2006). J. Alloys Compd. 415, 73-84]. In addition, the anisotropic atomic displacement parameters for all atomic sites have been determined for the first time. These compounds are confirmed to have the tetragonal $\mathrm{Zr}_{5} \mathrm{Si}_{4}$-type structure (space group: $P 4_{1} 2_{1} 2$ ), as reported previously (Smith et al., 1967). The structure is built up by distorted bodycentered cubes consisting of $\operatorname{Pr}(\mathrm{Nd})$ atoms, which are linked to each other by edge-sharing to form a three-dimensional framework. This framework delimits zigzag channels in which the silicon dimers are situated.

\section{Chemical context}

In natural science, there are some essential concepts concerned with symmetry, among which chiral symmetry is one of the fundamentals in all fields of physics, especially magnetism in solid-state materials. A chiral magnet in solids is of great interest in both science and technology. These magnets have been studied for novel phenomena such as chiral magnetic soliton lattices and use in future spintronic devices such as magnetic memories and logic gates. The critical point is that the crystal-structure chirality affects the arrangement of magnetic moments in these materials. The symmetry of crystals plays an important role in the spatial arrangement of the magnetic moments. For example, the intermetallic compound $\mathrm{YbNi}_{3} \mathrm{Al}_{9}$ has a trigonal $\mathrm{ErNi}_{3} \mathrm{Al}_{9}$ type structure in space group R32, a member of the Sohncke group (Gladyshevskii et al., 1993). This compound exhibits a characteristic helical magnetic structure, reflecting the symmetry of the crystal (Aoki et al., 2018). To study magnetism for chiral symmetry, we focused on the intermetallic compound $R_{5} \mathrm{Si}_{4}(R=\operatorname{Pr}$ and $\mathrm{Nd})$, which has a 
Table 1

Selected bond lengths $(\AA)$ for $\operatorname{Pr}_{5} \mathrm{Si}_{4}$.

\begin{tabular}{llll}
\hline $\operatorname{Pr} 1-\operatorname{Pr} 2^{\mathrm{i}}$ & $3.4914(4)$ & $\operatorname{Pr} 1-\mathrm{Si} 1^{\mathrm{ix}}$ & $3.1756(13)$ \\
\hline $\operatorname{Pr} 1-\operatorname{Pr} 2^{\text {ii }}$ & $3.5319(4)$ & $\operatorname{Pr} 1-\mathrm{Si} 2^{\mathrm{ii}}$ & $3.1780(13)$ \\
$\operatorname{Pr} 1-\operatorname{Pr} 2^{\text {iii }}$ & $3.5319(4)$ & $\operatorname{Pr} 1-\mathrm{Si} 2^{\mathrm{iii}}$ & $3.1780(13)$ \\
$\operatorname{Pr} 1-\operatorname{Pr} 2^{\text {iv }}$ & $3.4914(4)$ & $\operatorname{Pr} 2-\operatorname{Pr} 2^{\mathrm{i}}$ & $3.9561(6)$ \\
$\operatorname{Pr} 1-\operatorname{Pr} 3^{\mathrm{v}}$ & $3.6423(3)$ & $\operatorname{Pr} 2-\operatorname{Pr} 3^{\mathrm{vii}}$ & $3.9414(4)$ \\
$\operatorname{Pr} 1-\operatorname{Pr} 3^{\mathrm{vi}}$ & $3.6423(3)$ & $\operatorname{Pr} 2-\operatorname{Pr} 3^{x}$ & $3.9717(3)$ \\
$\operatorname{Pr} 1-\mathrm{Si} 1^{\text {vii }}$ & $3.1756(13)$ & $\operatorname{Pr} 2-\operatorname{Pr} 3^{\mathrm{xi}}$ & $3.9156(3)$ \\
$\operatorname{Pr} 1-\mathrm{Si} 1^{\text {viii }}$ & $3.0985(13)$ & $\operatorname{Pr} 3-\operatorname{Pr} 3^{\mathrm{ii}}$ & $4.0074(2)$ \\
$\operatorname{Pr} 1-\mathrm{Si} 1$ & $3.0985(13)$ & $\operatorname{Si} 1-\mathrm{Si} 2$ & $2.4738(16)$ \\
\hline
\end{tabular}

Symmetry codes: (i) $-y+1,-x+1,-z+\frac{1}{2}$; (ii) $-y+\frac{3}{2}, x+\frac{1}{2}, z+\frac{1}{4}$; (iii) $x+\frac{1}{2},-y+\frac{3}{2},-z+\frac{3}{4}$; (iv) $-x+1,-y+1, z+\frac{1}{2}$; (v) $-y+2,-x+1,-z+\frac{1}{2}$; (vi) $-x+1,-y+2, z+\frac{1}{2}$; (vii) $-y+\frac{3}{2}$, $x-\frac{1}{2}, z+\frac{1}{4}$; (viii) $y, x,-z+1$; (ix) $x-\frac{1}{2},-y+\frac{3}{2},-z+\frac{3}{4}$.

Table 2

Selected bond lengths $(\AA)$ for $\mathrm{Nd}_{5} \mathrm{Si}_{4}$.

\begin{tabular}{llll}
\hline $\mathrm{Nd} 1-\mathrm{Nd} 2^{\mathrm{i}}$ & $3.4725(5)$ & $\mathrm{Nd} 1-\mathrm{Si}^{\mathrm{ix}}$ & $3.1528(16)$ \\
\hline $\mathrm{Nd} 1-\mathrm{Nd} 2^{\mathrm{ii}}$ & $3.5021(5)$ & $\mathrm{Nd} 1-\mathrm{Si}^{\mathrm{ii}}$ & $3.1661(16)$ \\
$\mathrm{Nd} 1-\mathrm{Nd} 2^{\mathrm{iii}}$ & $3.5021(5)$ & $\mathrm{Nd} 1-\mathrm{Si} 2^{\mathrm{iii}}$ & $3.1661(16)$ \\
$\mathrm{Nd} 1-\mathrm{Nd} 2^{\mathrm{iv}}$ & $3.4725(5)$ & $\mathrm{Nd} 2-\mathrm{Nd} 2^{\mathrm{i}}$ & $3.9202(7)$ \\
$\mathrm{Nd} 1-\mathrm{Nd} 3^{\mathrm{v}}$ & $3.6265(3)$ & $\mathrm{Nd} 2-\mathrm{Nd} 3^{x}$ & $3.9094(4)$ \\
$\mathrm{Nd} 1-\mathrm{Nd} 3^{\mathrm{vi}}$ & $3.6265(3)$ & $\mathrm{Nd} 2-\mathrm{Nd} 3^{\mathrm{xi}}$ & $3.9378(4)$ \\
$\mathrm{Nd} 1-\mathrm{Si}^{\mathrm{vii}}$ & $3.1528(16)$ & $\mathrm{Nd} 2-\mathrm{Nd} 3^{\mathrm{vii}}$ & $3.9061(4)$ \\
$\mathrm{Nd} 1-\mathrm{Si} 1^{\text {viii }}$ & $3.0744(15)$ & $\mathrm{Nd} 3-\mathrm{Nd} 3^{\mathrm{xii}}$ & $3.9752(2)$ \\
$\mathrm{Nd} 1-\mathrm{Si} 1$ & $3.0744(15)$ & $\mathrm{Si} 1-\mathrm{Si} 2$ & $2.482(2)$ \\
\hline
\end{tabular}

Symmetry codes: (i) $-y+1,-x+1,-z+\frac{1}{2}$; (ii) $-y+\frac{3}{2}, x+\frac{1}{2}, z+\frac{1}{4}$; (iii) $x+\frac{1}{2},-y+\frac{3}{2},-z+\frac{3}{4}$; (iv) $-x+1,-y+1, z+\frac{1}{2}$; (v) $-y+2,-x+1,-z+\frac{1}{2}$; (vi) $-x+1,-y+2, z+\frac{1}{2}$; (vii) $-y+\frac{3}{2}$, $x-\frac{1}{2}, z+\frac{1}{4}$; (viii) $y, x,-z+1$; (ix) $x-\frac{1}{2},-y+\frac{3}{2},-z+\frac{3}{4}$.

tetragonal $\mathrm{Zr}_{5} \mathrm{Si}_{4}$-type crystal structure in the chiral space group $P 4_{1} 2_{1} 2$ (Smith et al., 1967).

Roger et al. (2006) isolated a small single crystal of $\mathrm{Nd}_{5} \mathrm{Si}_{4}$ by crushing the solidified sample and collected single-crystal X-ray data. Very recently, Sato et al. (2018) reported the single-crystal growth and magnetic properties of $\mathrm{Ce}_{5} \mathrm{Si}_{4}$, which has the same crystal structure as $\operatorname{Pr}_{5} \mathrm{Si}_{4}$ and $\mathrm{Nd}_{5} \mathrm{Si}_{4}$. At present, there has only been a report of large-size single-crystal growth for $R=\mathrm{Ce}$, and there are no reports of a large single crystal having been grown successfully for $R=\operatorname{Pr}$ or $\mathrm{Nd}$. In particular,
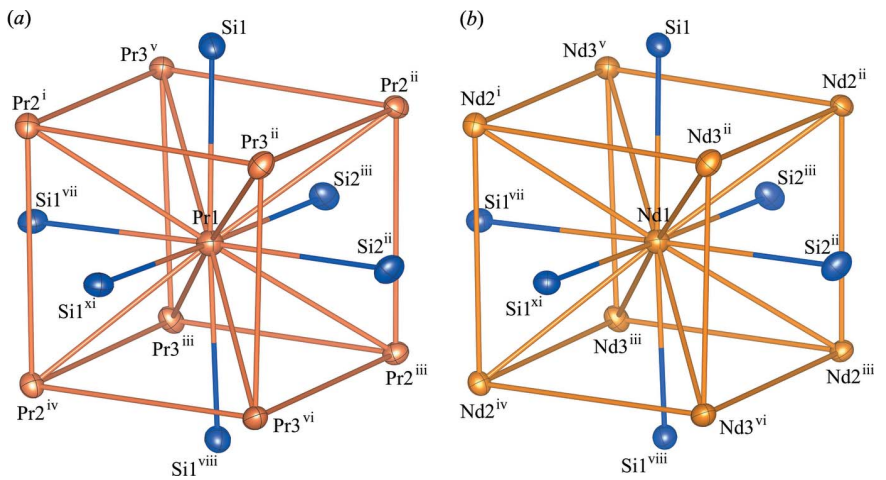

Figure 1

Principal units in the structure of $(a) \mathrm{Pr}_{5} \mathrm{Si}_{4}$ and $(b) \mathrm{Nd}_{5} \mathrm{Si}_{4}$, illustrated using VESTA (Momma \& Izumi, 2011). Displacement ellipsoids are drawn at the $90 \%$ probability level. Symmetry codes: (i) $-y+1,-x+1$, $-z+\frac{1}{2}$; (ii) $-y+\frac{3}{2}, x+\frac{1}{2}, z+\frac{1}{4}$; (iii) $x+\frac{1}{2},-y+\frac{3}{2},-z+\frac{3}{4}$; (iv) $-x+1,-y+1$, $z+\frac{1}{2} ;$ (v) $-y+2,-x+1,-z+\frac{1}{2} ;(\mathrm{vi})-x+1,-y+2, z+\frac{1}{2} ;$ (vii) $-y+\frac{3}{2}, x-\frac{1}{2}$, $z+\frac{1}{4}$; (viii) $y, x,-z+1$; (ix) $x-\frac{1}{2},-y+\frac{3}{2},-z+\frac{3}{4}$. for $\mathrm{Pr}_{5} \mathrm{Si}_{4}$, the crystal-structure analysis is based only on powder XRD data (Yang et al., 2002a,b,c, 2003; Cadogan et al., 2002; Smith et al., 1967). It is still unknown, however, whether there is a relationship between chiral symmetry and electronic properties, including magnetic ones. In this paper, we report the details of crystallographic studies of single-crystal X-ray analysis of high-quality single-crystalline $\mathrm{Pr}_{5} \mathrm{Si}_{4}$ and $\mathrm{Nd}_{5} \mathrm{Si}_{4}$, which are expected to be candidate materials for chiral magnets.

\section{Structural commentary}

The crystal structures of $\operatorname{Pr}_{5} \mathrm{Si}_{4}$ and $\mathrm{Nd}_{5} \mathrm{Si}_{4}$ refined in this study are essentially the same as those determined previously, belonging to chiral space group $P 4_{1} 2_{1} 2$ (No. 92) for $R=\mathrm{La}, \mathrm{Ce}$, and Nd (Yang et al., 2002a; Sato et al., 2018). The asymmetric unit of these compounds consists of three $\operatorname{Pr}(\mathrm{Nd})$ and two $\mathrm{Si}$ atoms. The $\operatorname{Pr} 1(\mathrm{Nd} 1)$ atom occupies the Wyckoff $4 a$ site, and the $\operatorname{Pr} 2(\mathrm{Nd} 2), \operatorname{Pr} 3(\mathrm{Nd} 3), \mathrm{Si} 1$ and $\mathrm{Si} 2$ are located on the general position $8 b$ sites. The principal units in the crystal structures of $\mathrm{Pr}_{5} \mathrm{Si}_{4}$ and $\mathrm{Nd}_{5} \mathrm{Si}_{4}$ are illustrated in Fig. 1, and selected bond lengths are given in Tables 1 and 2. The $\operatorname{Pr} 1(\mathrm{Nd} 1)$ coordination environment in these compounds can be described as a distorted cube with four $\operatorname{Pr} 2(\mathrm{Nd} 2)$ and four Pr3 (Nd3) [Pr1 - Pr2 and Pr1 - Pr3 bond lengths ranging from 3.4914 (4) to 3.6423 (3) $\AA$, Pr2 - Pr3 bond lengths in the range 3.9156 (3) to 4.0074 (2) $\AA, \mathrm{Nd} 1-\mathrm{Nd} 2$ and $\mathrm{Nd} 1-\mathrm{Nd} 3$ bond

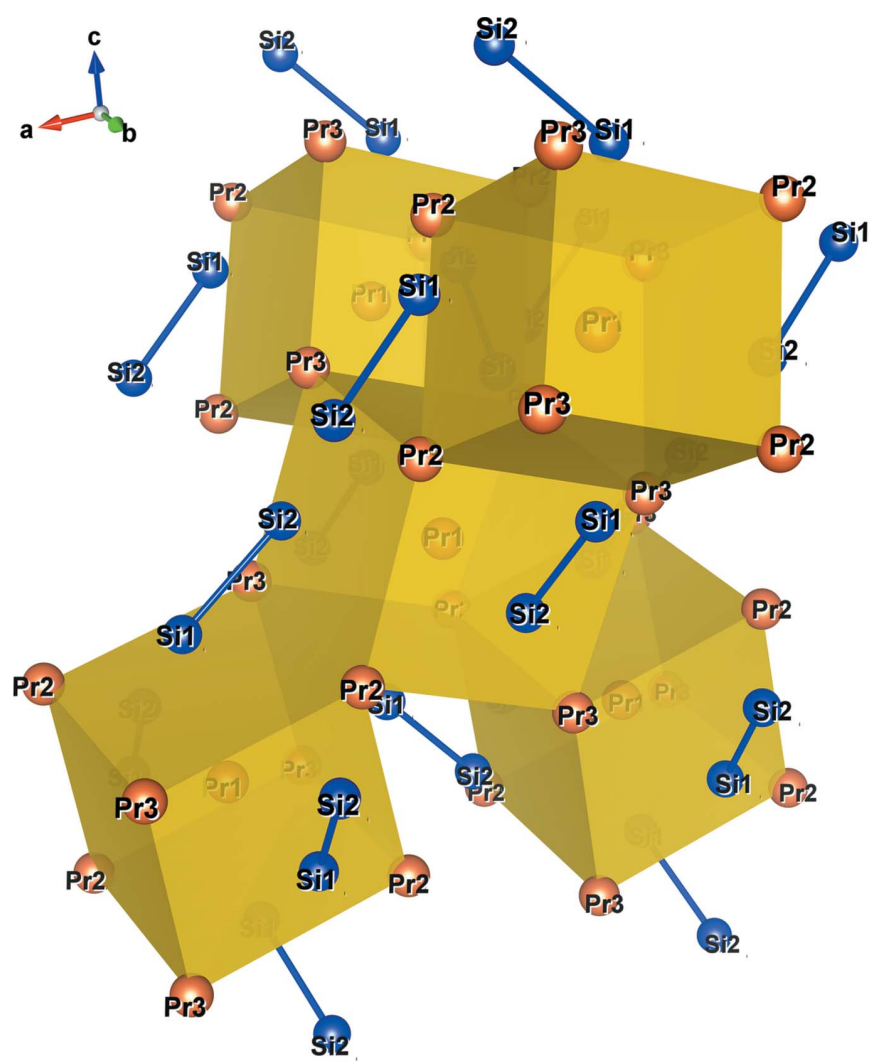

Figure 2

Parts of the crystal structure showing five distorted body-centered cubes sharing Pr2-Pr3 edges (polyhedral drawing). Si1 and Si2 atoms form dimers with atoms $\mathrm{Si} 2$ and Si1, respectively, of the adjacent unit. 
lengths of $3.4725(5)-3.6265(3) \AA$ and $\mathrm{Nd} 2-\mathrm{Nd} 3$ bond lengths of 3.9094 (4)-3.9752 (2) $\mathrm{]}$. In addition, the $\operatorname{Pr} 1(\mathrm{Nd} 1)-\mathrm{Si}$ bonds protruding through the distorted rectangular faces formed by two $\operatorname{Pr} 2(\mathrm{Nd} 2)$ and two $\operatorname{Pr} 3(\mathrm{Nd} 3)$ atoms have $\operatorname{Pr} 1-\mathrm{Si}$ bond lengths ranging from 3.0985 (13) to 3.1780 (13) $\AA$ and Nd1-Si bond lengths from 3.0744 (15) to 3.1661 (16) $\AA$. The distorted cubes are connected through common two $\operatorname{Pr} 2-\operatorname{Pr} 3(\mathrm{Nd} 2-\mathrm{Nd} 3)$ edges, and Si1 (Si2) atoms form dimers with Si2 (Si1) atoms in the adjacent unit (Fig. 2). The Si1 - Si2 bond length in $\mathrm{Pr}_{5} \mathrm{Si}_{4}$ is 2.4738 (16) $\AA$, and that of $\mathrm{Nd}_{5} \mathrm{Si}_{4}$ is $2.482(2) \AA$. The extended structure is shown in polyhedral representation in Fig. 3. The structure is built up by distorted body-centered cubes consisting of $\operatorname{Pr}(\mathrm{Nd})$ atoms, which are linked to each other by edge-sharing to form a three-dimensional framework. This framework delimits zigzag channels oriented along the [100] and [010] directions, in which the Si-Si dimers are situated.

\section{Synthesis and crystallization}

We have succeeded in growing single-crystalline samples of $\mathrm{Pr}_{5} \mathrm{Si}_{4}$ for the first time. For $\mathrm{Nd}_{5} \mathrm{Si}_{4}$, Roger et al. (2006) obtained a very small single crystal, but we have succeeded in growing a large single crystal. These compounds are incongruently melting compounds (Shukla et al., 2009), so we synthesized source materials with the non-stoichiometric

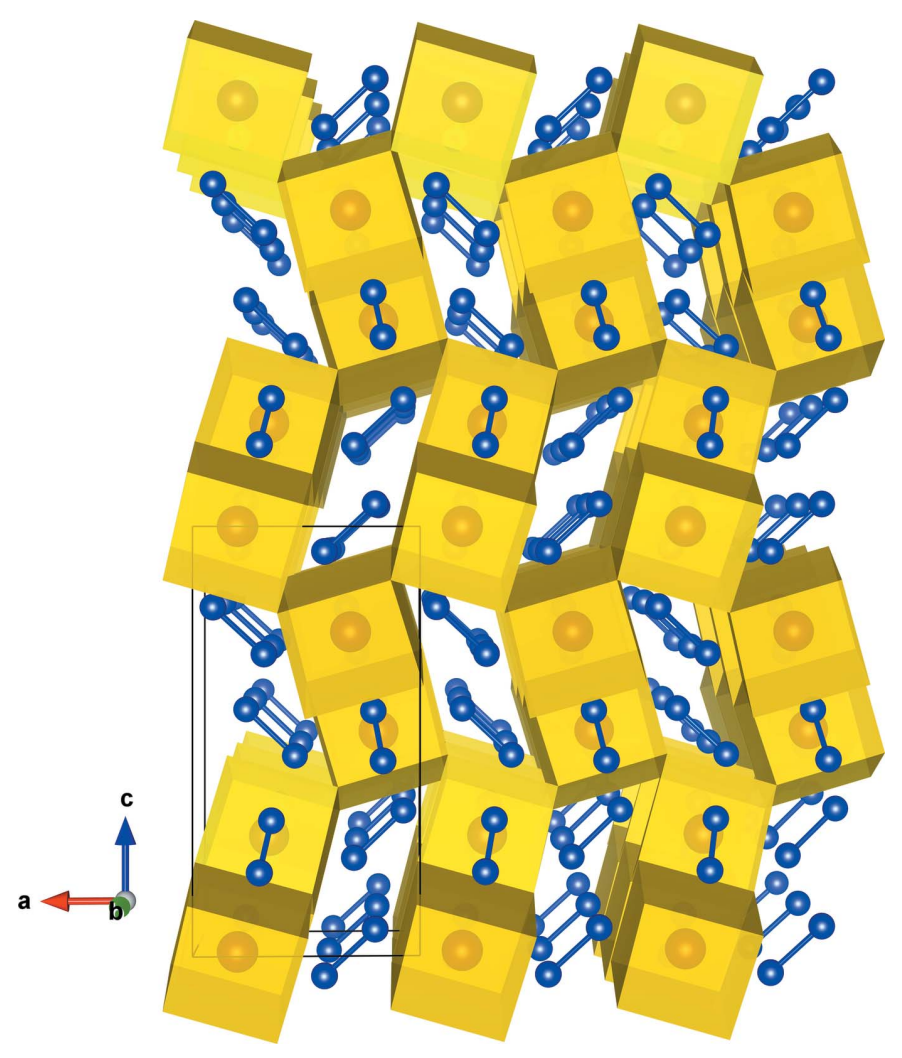

Figure 3

Polyhedral representation of the crystal structure of $\operatorname{Pr}_{5} \mathrm{Si}_{4}$ showing the $\mathrm{Si}-\mathrm{Si}$ dimers situated in zigzag channels running along the [100] and [010] directions. molar ratio of $\operatorname{Pr}(\mathrm{Nd})$ :Si of 58:42 in a mono-arc furnace. Each melted button of source materials was turned over and remelted three times to ensure homogeneity. Single crystals of $\mathrm{Pr}_{5} \mathrm{Si}_{4}$ and $\mathrm{Nd}_{5} \mathrm{Si}_{4}$ were grown by the Czochralski pulling method in a tetra arc furnace in an argon atmosphere on a water-cooled copper hearth. A tungsten rod was used as a pulling axis with no seed crystal, and after optimizing the initial conditions of the growth, the crystal was pulled at a constant rate of $12 \mathrm{~mm}_{\text {hour }}^{-1}$. The sizes of the grown ingots were about $30 \mathrm{~mm}$ in length and $5 \mathrm{~mm}$ in diameter. The grown single-crystal samples were characterized by powder X-ray diffraction using a Rigaku MiniFlexII diffractometer with $\mathrm{Cu}$ $K \alpha$ radiation. The powder X-ray diffraction peaks can be well indexed based on the tetragonal $\mathrm{Zr}_{5} \mathrm{Si}_{4}$-type structure. In addition, it has been confirmed that the whole grown crystal is a single grain crystal by means of the back-reflection Laue method.

\section{Database survey}

A survey of the Inorganic Crystal Structure Database (ICSD; Belsky et al., 2002) for $\mathrm{Pr}_{5} \mathrm{Si}_{4}$ yielded three hits. In all three, it is reported that $\mathrm{Pr}_{5} \mathrm{Si}_{4}$ has a $\mathrm{Zr}_{5} \mathrm{Si}_{4}$-type structure (Smith et al., 1967; ICSD 649362; Yang et al., 2002b; ICSD 95099; Yang et al., 2003; ICSD 98352). On the other hand, for $\mathrm{Nd}_{5} \mathrm{Si}_{4}$, previous reports have shown that $\mathrm{Nd}_{5} \mathrm{Si}_{4}$ has two types of crystal structure, a $\mathrm{Sm}_{5} \mathrm{Ge}_{4}$ type (Raman, 1968; ICSD 645983; Roger et al., 2006; ICSD 154658 and 154659) and a $\mathrm{Zr}_{5} \mathrm{Si}_{4}$-type structure (Smith et al., 1967; ICSD 645939; Mokra et al., 1978; ICSD 645946; Eremenko et al., 1984; ICSD 600990; Yang et al., 2002a; ICSD 94987; Yang et al., 2002c; ICSD 190404; Cadogan et al., 2002; ICSD 190404). Roger et al. (2006) reported that $\mathrm{Sm}_{5} \mathrm{Ge}_{4}$-type $\mathrm{Nd}_{5} \mathrm{Si}_{4}$ could be obtained only with the addition of a tiny amount of boron of less than three at.\% in the initial mixture, and that when synthesized with $\mathrm{Nd}$ and $\mathrm{Si}$ alone, $\mathrm{Zr}_{5} \mathrm{Si}_{4}$-type $\mathrm{Nd}_{5} \mathrm{Si}_{4}$ was obtained.

\section{Refinement}

Crystal data, data collection, and structure refinement details are summarized in Table 3 . The highest and deepest remaining difference electron density features are located at $0.90 \AA$ from Pr2 and $1.08 \AA$ from $\operatorname{Pr} 3$ for $\operatorname{Pr}_{5} \mathrm{Si}_{4}$, and $0.74 \AA$ from Nd1 and $1.38 \AA$ from $\mathrm{Nd} 2$ for $\mathrm{Nd}_{5} \mathrm{Si}_{4}$. The absolute structures of the samples were well-defined in space group $P 4_{1} 2_{1} 2$ (No. 92), although the bulk samples possibly also contain the other enantiomer; space group $P 4_{3} 2_{1} 2$ (No. 96).

\section{Acknowledgements}

The authors gratefully thank the Instrumental Analysis Center of Yokohama National University for providing access to the single-crystal X-ray diffractometer. 
Table 3

Experimental details.

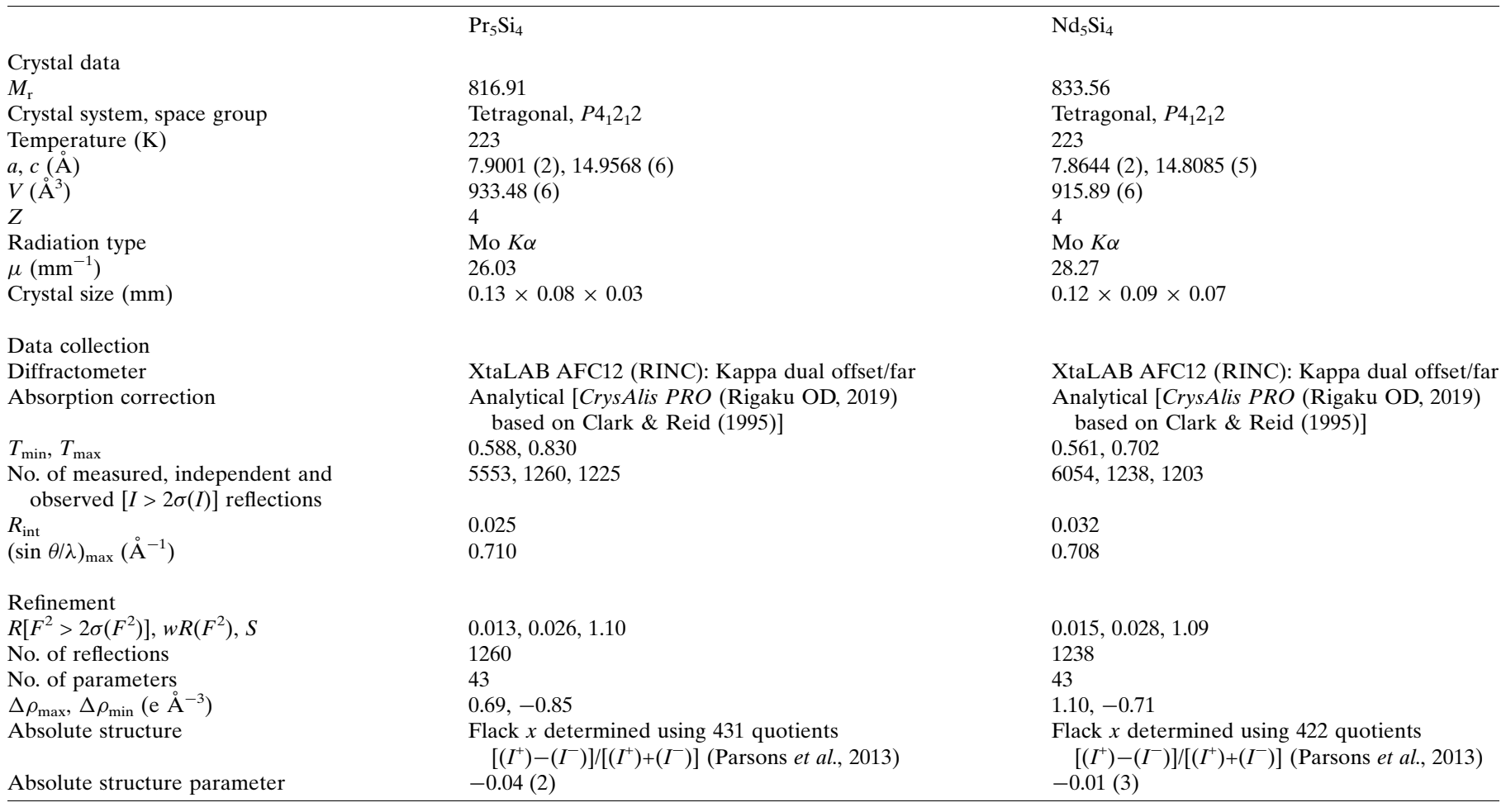

Computer programs: CrysAlis PRO (Rigaku OD, 2019), SHELXT (Sheldrick, 2015a), SHELXL (Sheldrick, 2015b), OLEX2 (Dolomanov et al., 2009) and publCIF (Westrip, 2010).

\section{Funding information}

Funding for this research was provided by: JSPS Grant-in-Aid for Scientific Research (KAKENHI) (grant No. 18K049922 and 18K03536).

\section{References}

Aoki, R., Togawa, Y. \& Ohara, S. (2018). Phys. Rev. B, 97, 214414. Belsky, A., Hellenbrandt, M., Karen, V. L. \& Luksch, P. (2002). Acta Cryst. B58, 364-369.

Cadogan, J. M., Ryan, D. H., Altounian, Z., Wang, H. B. \& Swainson, I. P. (2002). J. Phys. Condens. Matter, 14, 7191-7200.

Clark, R. C. \& Reid, J. S. (1995). Acta Cryst. A51, 887-897.

Dolomanov, O. V., Bourhis, L. J., Gildea, R. J., Howard, J. A. K. \& Puschmann, H. (2009). J. Appl. Cryst. 42, 339-341.

Eremenko, V. N., Meleshevich, K. A., Buyanov, Y. I. \& Obushenko, I. M. (1984). Dokl. Akad. Nauk Ukr. SSR, Ser. A, 11, 80.

Gladyshevskii, R. E., Cenzual, K., Flack, H. D. \& Parthé, E. (1993). Acta Cryst. B49, 468-474.

Mokra, I. R., Bodak, O. I. \& Gladyshevskii, E. I. (1978). Dopovidi Akademii Nauk Ukrains'koï RSR, Seriya A Fiziko-Mat. Tekh. Nauk, 1043-1045.

Momma, K. \& Izumi, F. (2011). J. Appl. Cryst. 44, 1272-1276.

Parsons, S., Flack, H. D. \& Wagner, T. (2013). Acta Cryst. B69, 249259.
Raman, A. (1968). Trans. Indian Inst. Met. 21, 5-8.

Rigaku OD (2019). CrysAlis PRO. Oxford Diffraction, Yarnton, England.

Roger, J., Babizhetskyy, V., Jardin, R., Halet, J. F. \& Guérin, R. (2006). J. Alloys Compd. 415, 73-84.

Sato, Y. J., Shimizu, Y., Nakamura, A., Homma, Y., Li, D., Maurya, A., Honda, F. \& Aoki, D. (2018). J. Phys. Soc. Jpn, 87, 074701.

Sheldrick, G. M. (2015a). Acta Cryst. A71, 3-8.

Sheldrick, G. M. (2015b). Acta Cryst. C71, 3-8.

Shukla, A., Kang, Y. B. \& Pelton, A. D. (2009). Int. J. Mater. Res. 100, 208-217.

Smith, G. S., Tharp, A. G. \& Johnson, W. (1967). Acta Cryst. 22, 940943.

Westrip, S. P. (2010). J. Appl. Cryst. 43, 920-925.

Yang, H. F., Rao, G. H., Chu, W. G., Liu, G. Y., Ouyang, Z. W. \& Liang, J. K. (2002a). J. Alloys Compd. 334, 131-134.

Yang, H. F., Rao, G. H., Chu, W. G., Liu, G. Y., Ouyang, Z. W. \& Liang, J. K. (2002b). J. Alloys Compd. 339, 189-194.

Yang, H. F., Rao, G. H., Liu, G. Y., Ouyang, Z. W., Liu, W. F., Feng, X. M., Chu, W. G. \& Liang, J. K. (2002c). J. Alloys Compd. 346, 190196.

Yang, H. F., Rao, G. H., Liu, G. Y., Ouyang, Z. W., Liu, W. F., Feng, X. M., Chu, W. G. \& Liang, J. K. (2003). J. Alloys Compd. 263, 146153. 


\section{supporting information}

Acta Cryst. (2020). E76, 510-513 [https://doi.org/10.1107/S2056989020002789]

Redetermination of the crystal structure of $R_{5} \mathrm{Si}_{4}(R=\mathrm{Pr}$, Nd) from single-crystal X-ray diffraction data

Kaori Yokota, Ryuta Watanuki, Miki Nakashima, Masatomo Uehara, Jun Gouchi, Yoshiya Uwatoko and Izuru Umehara

Computing details

For both structures, data collection: CrysAlis PRO (Rigaku OD, 2019); cell refinement: CrysAlis PRO (Rigaku OD, 2019); data reduction: CrysAlis PRO (Rigaku OD, 2019); program(s) used to solve structure: SHELXT (Sheldrick, 2015a); program(s) used to refine structure: SHELXL (Sheldrick, 2015b); molecular graphics: OLEX2 (Dolomanov et al., 2009); software used to prepare material for publication: publCIF (Westrip, 2010).

Pentapraseodymium tetrasiliside (A)

Crystal data

$\operatorname{Pr}_{5} \mathrm{Si}_{4}$

$M_{r}=816.91$

Tetragonal, $P 4_{1} 22_{1} 2$

Hall symbol: P 4abw 2nw

$a=7.9001(2) \AA$

$c=14.9568(6) \AA$

$V=933.48(6) \AA^{3}$

$Z=4$

$F(000)=1404$

Data collection

XtaLAB AFC12 (RINC): Kappa dual offset/far diffractometer

Radiation source: micro-focus sealed X-ray tube Mirror monochromator

Detector resolution: 5.8140 pixels $\mathrm{mm}^{-1}$

$\omega$ scans

Absorption correction: analytical

[CrysAlisPro (Rigaku OD, 2019) based on

Clark \& Reid (1995)]

Refinement

Refinement on $F^{2}$

Least-squares matrix: full

$R\left[F^{2}>2 \sigma\left(F^{2}\right)\right]=0.013$

$w R\left(F^{2}\right)=0.026$

$S=1.10$

1260 reflections

43 parameters

0 restraints
$D_{\mathrm{x}}=5.813 \mathrm{Mg} \mathrm{m}^{-3}$

Mo $K \alpha$ radiation, $\lambda=0.71073 \AA$

Cell parameters from 4009 reflections

$\theta=3.8-30.3^{\circ}$

$\mu=26.03 \mathrm{~mm}^{-1}$

$T=223 \mathrm{~K}$

Plate, metallic gray

$0.13 \times 0.08 \times 0.03 \mathrm{~mm}$

$T_{\min }=0.588, T_{\max }=0.830$

5553 measured reflections

1260 independent reflections

1225 reflections with $I>2 \sigma(I)$

$R_{\text {int }}=0.025$

$\theta_{\text {max }}=30.3^{\circ}, \theta_{\text {min }}=3.7^{\circ}$

$h=-11 \rightarrow 11$

$k=-10 \rightarrow 10$

$l=-19 \rightarrow 17$

Primary atom site location: dual

$w=1 /\left[\sigma^{2}\left(F_{\mathrm{o}}^{2}\right)+(0.0078 P)^{2}+0.0556 P\right]$

where $P=\left(F_{\mathrm{o}}^{2}+2 F_{\mathrm{c}}^{2}\right) / 3$

$(\Delta / \sigma)_{\max }=0.001$

$\Delta \rho_{\max }=0.69$ e $\AA^{-3}$

$\Delta \rho_{\min }=-0.85$ e $\AA^{-3}$

Extinction correction: SHELXL (Sheldrick, $2015 \mathrm{~b}), \mathrm{Fc}^{*}=\mathrm{kFc}\left[1+0.001 \times \mathrm{Fc}^{2} \lambda^{3} / \sin (2 \theta)\right]^{-1 / 4}$ 
Extinction coefficient: 0.00247 (9)

Special details

Geometry. All esds (except the esd in the dihedral angle between two 1.s. planes) are estimated using the full covariance matrix. The cell esds are taken into account individually in the estimation of esds in distances, angles and torsion angles; correlations between esds in cell parameters are only used when they are defined by crystal symmetry. An approximate (isotropic) treatment of cell esds is used for estimating esds involving l.s. planes.

Fractional atomic coordinates and isotropic or equivalent isotropic displacement parameters $\left(\AA^{2}\right)$

\begin{tabular}{lllll}
\hline & $x$ & $y$ & $z$ & $U_{\text {iso }} * / U_{\text {eq }}$ \\
\hline Pr1 & $0.81086(3)$ & $0.81086(3)$ & 0.500000 & $0.00721(8)$ \\
Pr2 & $0.51578(3)$ & $0.37028(3)$ & $0.12478(2)$ & $0.00578(7)$ \\
Pr3 & $0.51088(3)$ & $0.87144(3)$ & $0.04758(2)$ & $0.00676(7)$ \\
Si1 & $0.92537(16)$ & $0.71000(16)$ & $0.30916(8)$ & $0.0069(2)$ \\
Si2 & $0.69967(16)$ & $0.66478(16)$ & $0.19702(8)$ & $0.0078(2)$ \\
\hline
\end{tabular}

Atomic displacement parameters $\left(\AA^{2}\right)$

\begin{tabular}{lllllll}
\hline & $U^{11}$ & $U^{22}$ & $U^{33}$ & $U^{12}$ & $U^{13}$ & $U^{23}$ \\
\hline Pr1 & $0.00720(10)$ & $0.00720(10)$ & $0.00723(16)$ & $-0.00017(11)$ & $0.00069(9)$ & $-0.00069(9)$ \\
Pr2 & $0.00587(11)$ & $0.00618(12)$ & $0.00529(12)$ & $-0.00033(9)$ & $0.00050(9)$ & $-0.00083(8)$ \\
Pr3 & $0.00630(12)$ & $0.00650(12)$ & $0.00748(12)$ & $-0.00045(7)$ & $-0.00114(9)$ & $0.00172(9)$ \\
Si1 & $0.0074(5)$ & $0.0078(6)$ & $0.0056(6)$ & $-0.0011(4)$ & $-0.0004(4)$ & $0.0001(5)$ \\
Si2 & $0.0066(5)$ & $0.0074(6)$ & $0.0094(6)$ & $-0.0018(4)$ & $-0.0012(5)$ & $0.0007(5)$ \\
\hline
\end{tabular}

Geometric parameters $\left(\hat{A},{ }^{\circ}\right)$

\begin{tabular}{|c|c|c|c|}
\hline $\operatorname{Pr} 1-\operatorname{Pr} 2^{\mathrm{i}}$ & $3.4914(4)$ & $\operatorname{Pr} 2-\operatorname{Pr} 3^{x i}$ & $3.9156(3)$ \\
\hline $\operatorname{Pr} 1-\operatorname{Pr} 2^{\mathrm{ii}}$ & $3.5319(4)$ & $\operatorname{Pr} 2-\mathrm{Si}^{\mathrm{xi}}$ & $3.0641(13)$ \\
\hline $\operatorname{Pr} 1-\operatorname{Pr} 2^{\mathrm{iii}}$ & $3.5319(4)$ & $\operatorname{Pr} 2-\mathrm{Si}^{\mathrm{i}}$ & $3.1005(14)$ \\
\hline $\operatorname{Pr} 1-\operatorname{Pr} 2^{\mathrm{iv}}$ & $3.4914(4)$ & $\operatorname{Pr} 2-\mathrm{Si}^{\mathrm{xii}}$ & $3.0668(14)$ \\
\hline $\operatorname{Pr} 1-\operatorname{Pr} 3^{v}$ & $3.6423(3)$ & $\mathrm{Pr} 2-\mathrm{Si} 2$ & $2.9480(13)$ \\
\hline $\operatorname{Pr} 1-\operatorname{Pr} 3^{\mathrm{vi}}$ & $3.6423(3)$ & $\operatorname{Pr} 2-\mathrm{Si}^{\mathrm{xi}}$ & $2.9737(13)$ \\
\hline $\operatorname{Pr} 1-\mathrm{Si}_{1}^{\mathrm{vii}}$ & $3.1756(13)$ & $\operatorname{Pr} 2-\mathrm{Si} 2^{\mathrm{i}}$ & $3.0730(13)$ \\
\hline $\operatorname{Pr} 1-\mathrm{Si1}^{\mathrm{viii}}$ & 3.0985 (13) & $\operatorname{Pr} 3-\operatorname{Pr} 3^{\mathrm{ii}}$ & $4.0074(2)$ \\
\hline $\operatorname{Pr} 1-\mathrm{Si} 1$ & $3.0985(13)$ & $\operatorname{Pr} 3-\mathrm{Si}^{\mathrm{xiii}}$ & $3.1554(13)$ \\
\hline $\operatorname{Pr} 1-\mathrm{Si}^{\mathrm{ix}}$ & $3.1756(13)$ & $\operatorname{Pr} 3-\mathrm{Si}^{\mathrm{xii}}$ & $3.3434(12)$ \\
\hline $\operatorname{Pr} 1-\mathrm{Si} 2^{\mathrm{ii}}$ & $3.1780(13)$ & $\operatorname{Pr} 3-\mathrm{Si}^{\mathrm{xiv}}$ & $3.1957(12)$ \\
\hline $\operatorname{Pr} 1-\mathrm{Si} 2^{\mathrm{iii}}$ & $3.1780(13)$ & $\operatorname{Pr} 3-\mathrm{Si} 2^{\mathrm{xiii}}$ & $3.2566(12)$ \\
\hline $\operatorname{Pr} 2-\operatorname{Pr} 2^{i}$ & $3.9561(6)$ & $\operatorname{Pr} 3-\mathrm{Si}^{\mathrm{xii}}$ & $3.1708(12)$ \\
\hline $\operatorname{Pr} 2-\operatorname{Pr} 3^{\text {vii }}$ & $3.9414(4)$ & $\mathrm{Pr} 3-\mathrm{Si} 2$ & $3.1442(12)$ \\
\hline $\operatorname{Pr} 2-\operatorname{Pr} 3^{x}$ & $3.9717(3)$ & $\mathrm{Si} 1-\mathrm{Si} 2$ & $2.4738(16)$ \\
\hline $\operatorname{Pr} 2^{\mathrm{i}}-\operatorname{Pr} 1-\operatorname{Pr} 2^{\mathrm{iv}}$ & $71.331(11)$ & $\mathrm{Si} 2^{\mathrm{xi}}-\mathrm{Pr} 2-\mathrm{Si} 2^{\mathrm{i}}$ & $124.47(4)$ \\
\hline 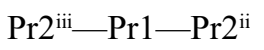 & $68.120(11)$ & $\operatorname{Pr} 1^{\mathrm{xvi}}-\operatorname{Pr} 3-\operatorname{Pr} 1^{\mathrm{xii}}$ & $97.461(6)$ \\
\hline
\end{tabular}




\begin{tabular}{|c|c|}
\hline $\operatorname{Pr} 2^{\mathrm{iv}}-\operatorname{Pr} 1-\operatorname{Pr} 2^{\mathrm{ii}}$ & $176.841(6)$ \\
\hline $\operatorname{Pr} 2^{\mathrm{i}}-\operatorname{Pr} 1-\operatorname{Pr} 2^{\mathrm{iii}}$ & $176.841(6)$ \\
\hline $\operatorname{Pr} 2^{\mathrm{i}}-\operatorname{Pr} 1-\operatorname{Pr} 2^{\mathrm{ii}}$ & $110.343(4)$ \\
\hline $\operatorname{Pr} 2^{\mathrm{iv}}-\operatorname{Pr} 1-\operatorname{Pr} 2^{\mathrm{iii}}$ & $110.343(4)$ \\
\hline $\operatorname{Pr} 2^{\mathrm{iv}}-\operatorname{Pr} 1-\operatorname{Pr} 3^{\mathrm{v}}$ & $109.131(9)$ \\
\hline $\operatorname{Pr} 2^{\mathrm{iii}}-\operatorname{Pr} 1-\operatorname{Pr} 3^{\mathrm{vi}}$ & $74.025(6)$ \\
\hline $\operatorname{Pr} 2^{i}-\operatorname{Pr} 1-\operatorname{Pr} 3^{v}$ & $70.258(6)$ \\
\hline $\operatorname{Pr} 2^{\mathrm{ii}}-\operatorname{Pr} 1-\operatorname{Pr} 3^{\mathrm{v}}$ & $74.025(6)$ \\
\hline $\operatorname{Pr} 2^{\mathrm{ii}}-\operatorname{Pr} 1-\operatorname{Pr} 3^{\mathrm{vi}}$ & $106.588(8)$ \\
\hline $\operatorname{Pr} 2^{\mathrm{i}}-\operatorname{Pr} 1-\operatorname{Pr} 3^{\mathrm{vi}}$ & $109.131(9)$ \\
\hline $\operatorname{Pr} 2^{\mathrm{iv}}-\operatorname{Pr} 1-\operatorname{Pr} 3^{\mathrm{vi}}$ & $70.258(6)$ \\
\hline $\operatorname{Pr} 2^{\mathrm{iii}-\operatorname{Pr} 1-\operatorname{Pr} 3^{\mathrm{v}}}$ & $106.588(8)$ \\
\hline $\operatorname{Pr} 3^{\mathrm{vi}}-\operatorname{Pr} 1-\operatorname{Pr} 3^{\mathrm{v}}$ & $179.290(14)$ \\
\hline Si1-Pr1—Pr $2^{\mathrm{iii}}$ & $122.55(3)$ \\
\hline Si $1^{\text {viii }-P r 1}-\operatorname{Pr} 2^{i}$ & $127.07(3)$ \\
\hline Si1-Pr1-Pr2 ${ }^{\mathrm{ii}}$ & $54.63(3)$ \\
\hline $\operatorname{Si} 1^{\mathrm{ix}}-\operatorname{Pr} 1-\operatorname{Pr} 2^{\mathrm{iii}}$ & $128.63(2)$ \\
\hline $\mathrm{Si} 1^{\mathrm{ix}}-\operatorname{Pr} 1-\operatorname{Pr} 2^{\mathrm{iv}}$ & $54.47(2)$ \\
\hline $\operatorname{Si} 1-\operatorname{Pr} 1-\operatorname{Pr} 2^{i}$ & $55.75(3)$ \\
\hline $\mathrm{Si} 1^{\mathrm{vii}}-\operatorname{Pr} 1-\operatorname{Pr} 2^{\mathrm{iv}}$ & $54.52(2)$ \\
\hline $\mathrm{Si} 1^{\mathrm{viii}}-\operatorname{Pr} 1-\operatorname{Pr} 2^{\mathrm{iii}}$ & $54.63(3)$ \\
\hline $\mathrm{Si} 1^{\mathrm{ix}}-\operatorname{Pr} 1-\operatorname{Pr} 2^{\mathrm{i}}$ & $54.52(2)$ \\
\hline $\mathrm{Si} 1^{\mathrm{vii}}-\operatorname{Pr} 1-\operatorname{Pr} 2^{\mathrm{iii}}$ & $124.06(2)$ \\
\hline $\mathrm{Si}^{\mathrm{vii}}-\operatorname{Pr} 1-\operatorname{Pr} 2^{\mathrm{i}}$ & $54.47(2)$ \\
\hline Si1-Pr1-Pr2 $2^{\mathrm{iv}}$ & $127.07(3)$ \\
\hline $\mathrm{Si} 1^{\mathrm{viii}}-\operatorname{Pr} 1-\operatorname{Pr} 2^{\mathrm{ii}}$ & $122.55(3)$ \\
\hline $\mathrm{Si}^{\mathrm{viii}}-\operatorname{Pr} 1-\operatorname{Pr} 2^{\mathrm{iv}}$ & $55.75(3)$ \\
\hline $\mathrm{Si} 1^{\mathrm{ix}}-\operatorname{Pr} 1-\operatorname{Pr} 2^{\mathrm{ii}}$ & $124.06(2)$ \\
\hline 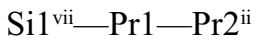 & $128.63(2)$ \\
\hline Sil-Pr1-Pr3 $3^{\mathrm{vi}}$ & $124.12(2)$ \\
\hline $\mathrm{Si}^{\mathrm{viii}}-\operatorname{Pr} 1-\operatorname{Pr} 3^{\mathrm{v}}$ & $124.12(2)$ \\
\hline $\mathrm{Si}^{\mathrm{vii}}-\operatorname{Pr} 1-\operatorname{Pr} 3^{\mathrm{v}}$ & $54.62(2)$ \\
\hline $\mathrm{Si}^{\mathrm{vii}}-\operatorname{Pr} 1-\operatorname{Pr} 3^{\mathrm{vi}}$ & $55.90(2)$ \\
\hline $\mathrm{Si} 1^{\mathrm{ix}}-\operatorname{Pr} 1-\operatorname{Pr} 3^{\mathrm{v}}$ & $124.76(3)$ \\
\hline $\mathrm{Si} 1^{\mathrm{ix}}-\operatorname{Pr} 1-\operatorname{Pr} 3^{\mathrm{vi}}$ & $54.62(2)$ \\
\hline $\mathrm{Si}^{\mathrm{vii}}-\operatorname{Pr} 1-\operatorname{Pr} 3^{\mathrm{vi}}$ & $124.76(3)$ \\
\hline Si1-Pr1-Pr3 ${ }^{v}$ & $55.90(2)$ \\
\hline $\mathrm{Si} 1-\operatorname{Pr} 1-\mathrm{Si}^{\mathrm{vii}}$ & $91.45(4)$ \\
\hline 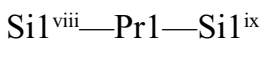 & $91.45(4)$ \\
\hline Sil-Pr1-Si1 ${ }^{\mathrm{ix}}$ & $90.57(2)$ \\
\hline $\mathrm{Si} 1^{\mathrm{vii}}-\operatorname{Pr} 1-\mathrm{Si} 1^{\mathrm{vii}}$ & $90.57(2)$ \\
\hline Si1-Pr1-Si1 $1^{\text {viii }}$ & $177.18(5)$ \\
\hline $\mathrm{Si} 1^{\mathrm{ix}}-\operatorname{Pr} 1-\mathrm{Si} 1^{\mathrm{vii}}$ & $88.74(5)$ \\
\hline 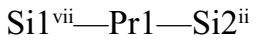 & $178.20(3)$ \\
\hline $\mathrm{Si} 1^{\mathrm{ix}}-\operatorname{Pr} 1-\mathrm{Si} 2^{\mathrm{ii}}$ & $92.22(3)$ \\
\hline $\mathrm{Si} 1^{\mathrm{vii}}-\operatorname{Pr} 1-\mathrm{Si} 2^{\mathrm{iii}}$ & $92.22(3)$ \\
\hline $\mathrm{Si} 1-\operatorname{Pr} 1-\mathrm{Si} 2^{\mathrm{ii}}$ & $90.07(3)$ \\
\hline $\mathrm{Si} 1^{\mathrm{ix}}-\operatorname{Pr} 1-\mathrm{Si} 2^{\mathrm{iii}}$ & $178.20(3)$ \\
\hline
\end{tabular}

\begin{tabular}{|c|c|}
\hline $\operatorname{Pr} 1^{x v i}-\operatorname{Pr} 3-\operatorname{Pr} 2^{x}$ & $99.525(8)$ \\
\hline $\operatorname{Pr} 1^{x i i}-\operatorname{Pr} 3-\operatorname{Pr} 2^{x v i i}$ & $158.527(9)$ \\
\hline $\operatorname{Pr} 1^{x v i}-\operatorname{Pr} 3-\operatorname{Pr} 2^{x i i}$ & $136.554(9)$ \\
\hline $\operatorname{Pr} 1^{x i i}-\operatorname{Pr} 3-\operatorname{Pr} 2^{x}$ & $97.821(7)$ \\
\hline $\operatorname{Pr} 1^{\mathrm{xii}}-\operatorname{Pr} 3-\operatorname{Pr} 2^{\mathrm{xiii}}$ & $99.441(7)$ \\
\hline $\operatorname{Pr} 1^{x v i}-\operatorname{Pr} 3-\operatorname{Pr} 2^{x v i i}$ & $102.639(8)$ \\
\hline $\operatorname{Pr} 1^{\mathrm{xvi}}-\operatorname{Pr} 3-\operatorname{Pr} 3^{\mathrm{ii}}$ & $98.905(6)$ \\
\hline $\operatorname{Pr} 1^{\mathrm{xii}}-\operatorname{Pr} 3-\operatorname{Pr} 3^{\mathrm{ii}}$ & $56.190(4)$ \\
\hline $\operatorname{Pr} 2^{x i i}-\operatorname{Pr} 3-\operatorname{Pr} 2^{x v i i}$ & $60.464(9)$ \\
\hline $\operatorname{Pr} 2^{\mathrm{xvii}}-\operatorname{Pr} 3-\operatorname{Pr} 2^{\mathrm{x}}$ & $86.316(6)$ \\
\hline $\operatorname{Pr} 2^{x i i i}-\operatorname{Pr} 3-\operatorname{Pr} 2^{x}$ & $117.252(7)$ \\
\hline $\operatorname{Pr} 2^{x}-\operatorname{Pr} 3-\operatorname{Pr} 3^{\mathrm{ii}}$ & $149.882(8)$ \\
\hline $\operatorname{Pr} 2^{\mathrm{xvii}}-\operatorname{Pr} 3-\operatorname{Pr} 3^{\mathrm{ii}}$ & $112.494(8)$ \\
\hline $\operatorname{Pr} 2^{\mathrm{xiii}}-\operatorname{Pr} 3-\operatorname{Pr} 3^{\mathrm{ii}}$ & $60.158(7)$ \\
\hline $\mathrm{Si}^{\mathrm{xii}}-\operatorname{Pr} 3-\operatorname{Pr} 1^{\mathrm{xvi}}$ & $89.84(2)$ \\
\hline $\mathrm{Si}^{\mathrm{xiv}}-\operatorname{Pr} 3-\operatorname{Pr} 1^{\mathrm{xii}}$ & $54.18(2)$ \\
\hline $\mathrm{Si}^{\mathrm{xiii}}-\operatorname{Pr} 3-\operatorname{Pr} 1^{\mathrm{xvi}}$ & $55.14(2)$ \\
\hline $\mathrm{Si}^{\mathrm{xii}}-\operatorname{Pr} 3-\operatorname{Pr} 1^{\mathrm{xii}}$ & $51.84(2)$ \\
\hline Si1 $1^{\mathrm{xii}}-\operatorname{Pr} 3-\operatorname{Pr} 1^{\mathrm{xii}}$ & $144.17(3)$ \\
\hline $\mathrm{Si} 1^{\mathrm{xiv}}-\operatorname{Pr} 3-\operatorname{Pr} 1^{\mathrm{xvi}}$ & $53.40(2)$ \\
\hline $\mathrm{Si}^{\mathrm{xiii}}-\operatorname{Pr} 3-\operatorname{Pr} 2^{\mathrm{xii}}$ & $89.98(2)$ \\
\hline $\mathrm{Si}^{\mathrm{xiv}}-\operatorname{Pr} 3-\operatorname{Pr} 2^{\mathrm{xiii}}$ & $108.89(3)$ \\
\hline Si $1^{x i i}-\operatorname{Pr} 3-\operatorname{Pr} 2^{x v i i}$ & $134.80(2)$ \\
\hline $\operatorname{Si} 1^{\text {xiv }-P r 3-P r} 2^{x}$ & $129.63(3)$ \\
\hline Si1 $1^{\mathrm{xii}}-\operatorname{Pr} 3-\operatorname{Pr} 2^{\mathrm{xiii}}$ & $131.45(2)$ \\
\hline $\mathrm{Si}^{\mathrm{xiii}}-\operatorname{Pr} 3-\operatorname{Pr} 2^{\mathrm{xvii}}$ & $50.33(3)$ \\
\hline $\mathrm{Si} 1^{\mathrm{xii}}-\operatorname{Pr} 3-\operatorname{Pr} 2^{\mathrm{x}}$ & $48.60(2)$ \\
\hline $\mathrm{Si} 1^{\mathrm{xiii}}-\operatorname{Pr} 3-\operatorname{Pr} 2^{\mathrm{x}}$ & $108.41(2)$ \\
\hline $\mathrm{Si} 1^{\mathrm{xiv}}-\operatorname{Pr} 3-\operatorname{Pr} 2^{\mathrm{xvii}}$ & $136.01(3)$ \\
\hline $\mathrm{Si}^{\mathrm{xiii}}-\operatorname{Pr} 3-\operatorname{Pr} 3^{\mathrm{ii}}$ & $101.66(2)$ \\
\hline $\mathrm{Si} 1^{\mathrm{xii}}-\operatorname{Pr} 3-\operatorname{Pr} 3^{\mathrm{ii}}$ & $108.02(2)$ \\
\hline $\mathrm{Si} 1^{\mathrm{xiv}}-\operatorname{Pr} 3-\operatorname{Pr} 3^{\mathrm{ii}}$ & $50.43(2)$ \\
\hline Si1 $1^{\mathrm{xiv}}-\mathrm{Pr} 3-\mathrm{Si}^{\mathrm{xii}}$ & $85.949(17)$ \\
\hline Si1 $1^{\mathrm{xii}}-\mathrm{Pr} 3-\mathrm{Si}^{\mathrm{xii}}$ & $137.237(18)$ \\
\hline $\mathrm{Si} 1^{\mathrm{xii}}-\mathrm{Pr} 3-\mathrm{Si}^{\mathrm{xiv}}$ & $90.036(12)$ \\
\hline $\mathrm{Si} 1^{\mathrm{xiv}}-\mathrm{Pr} 3-\mathrm{Si} 2^{\mathrm{xiii}}$ & $90.54(3)$ \\
\hline $\mathrm{Si} 1^{\mathrm{xii}}-\mathrm{Pr} 3-\mathrm{Si} 2^{\mathrm{xiii}}$ & $45.35(3)$ \\
\hline Si1 $1^{\mathrm{xii}}-\mathrm{Pr} 3-\mathrm{Si} 2^{\mathrm{xii}}$ & $92.74(3)$ \\
\hline $\mathrm{Si} 2^{\mathrm{xii}}-\operatorname{Pr} 3-\operatorname{Pr} 1^{\mathrm{xii}}$ & $87.19(2)$ \\
\hline Si2-Pr3-Pr1 ${ }^{x i i}$ & $54.53(2)$ \\
\hline Si2-Pr3-Pr1 $1^{\mathrm{xvi}}$ & $145.87(2)$ \\
\hline $\mathrm{Si} 2^{\mathrm{xii}}-\operatorname{Pr} 3-\operatorname{Pr} 1^{\mathrm{xii}}$ & $123.76(2)$ \\
\hline $\mathrm{Si} 2^{\mathrm{xiii}}-\operatorname{Pr} 3-\operatorname{Pr} 1^{\mathrm{xvi}}$ & $90.26(2)$ \\
\hline $\mathrm{Si} 2^{\mathrm{xii}}-\operatorname{Pr} 3-\operatorname{Pr} 1^{\mathrm{xvi}}$ & $55.08(2)$ \\
\hline $\mathrm{Si} 2^{\mathrm{xiii}}-\operatorname{Pr} 3-\operatorname{Pr} 2^{\mathrm{xvii}}$ & $49.43(2)$ \\
\hline $\mathrm{Si} 2^{\mathrm{xiii}}-\operatorname{Pr} 3-\operatorname{Pr} 2^{\mathrm{xiii}}$ & $47.45(2)$ \\
\hline $\mathrm{Si} 2^{\mathrm{xii}}-\operatorname{Pr} 3-\operatorname{Pr} 2^{\mathrm{x}}$ & $135.71(2)$ \\
\hline $\mathrm{Si} 2^{\mathrm{xii}}-\operatorname{Pr} 3-\operatorname{Pr} 2^{\mathrm{xiii}}$ & $164.53(2)$ \\
\hline
\end{tabular}




\begin{tabular}{|c|c|}
\hline $\mathrm{Si} 1-\operatorname{Pr} 1-\mathrm{Si} 2^{\mathrm{iii}}$ & $87.88(3)$ \\
\hline $\mathrm{Si}^{\mathrm{viii}}-\operatorname{Pr} 1-\mathrm{Si} 2^{\mathrm{iii}}$ & $90.07(3)$ \\
\hline $\mathrm{Si} 1^{\mathrm{viii}}-\operatorname{Pr} 1-\mathrm{Si} 2^{\mathrm{ii}}$ & $87.88(3)$ \\
\hline 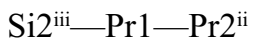 & $54.20(2)$ \\
\hline 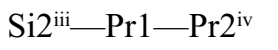 & $127.29(2)$ \\
\hline $\mathrm{Si} 2^{\mathrm{ii}}-\operatorname{Pr} 1-\operatorname{Pr} 2^{\mathrm{ii}}$ & $51.80(2)$ \\
\hline 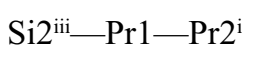 & $125.04(2)$ \\
\hline $\mathrm{Si} 2^{\mathrm{ii}}-\operatorname{Pr} 1-\operatorname{Pr} 2^{\mathrm{iv}}$ & $125.04(2)$ \\
\hline $\mathrm{Si} 2^{\mathrm{ii}}-\operatorname{Pr} 1-\operatorname{Pr} 2^{\mathrm{iii}}$ & $54.20(2)$ \\
\hline $\mathrm{Si} 2^{\mathrm{iii}}-\operatorname{Pr} 1-\operatorname{Pr} 2^{\mathrm{iii}}$ & $51.80(2)$ \\
\hline $\mathrm{Si} 2^{\mathrm{ii}}-\operatorname{Pr} 1-\operatorname{Pr} 2^{\mathrm{i}}$ & $127.29(2)$ \\
\hline $\mathrm{Si} 2^{\mathrm{iii}}-\operatorname{Pr} 1-\operatorname{Pr} 3^{\mathrm{vi}}$ & $125.73(2)$ \\
\hline $\mathrm{Si} 2^{\mathrm{ii}}-\operatorname{Pr} 1-\operatorname{Pr} 3^{\mathrm{vi}}$ & $54.90(2)$ \\
\hline $\mathrm{Si} 2^{\mathrm{ii}}-\operatorname{Pr} 1-\operatorname{Pr} 3^{\mathrm{v}}$ & $125.73(2)$ \\
\hline 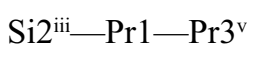 & $54.90(2)$ \\
\hline 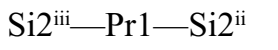 & $86.85(5)$ \\
\hline $\operatorname{Pr} 1^{x v}-\operatorname{Pr} 2-\operatorname{Pr} 1^{x i i}$ & $103.710(6)$ \\
\hline $\operatorname{Pr} 1^{\mathrm{xii}}-\operatorname{Pr} 2-\operatorname{Pr} 2^{\mathrm{i}}$ & $55.940(5)$ \\
\hline $\operatorname{Pr} 1^{x v}-\operatorname{Pr} 2-\operatorname{Pr} 2^{\mathrm{i}}$ & $140.135(10$ \\
\hline $\operatorname{Pr} 1^{\mathrm{xv}}-\operatorname{Pr} 2-\operatorname{Pr} 3^{\mathrm{vii}}$ & $102.847(8)$ \\
\hline $\operatorname{Pr} 1^{\mathrm{xv}}-\operatorname{Pr} 2-\operatorname{Pr} 3^{\mathrm{x}}$ & $106.888(8)$ \\
\hline $\operatorname{Pr} 1^{\mathrm{xii}}-\operatorname{Pr} 2-\operatorname{Pr} 3^{\mathrm{xi}}$ & $106.191(8)$ \\
\hline $\operatorname{Pr} 1^{\mathrm{xii}}-\operatorname{Pr} 2-\operatorname{Pr} 3^{\mathrm{vii}}$ & $105.646(8)$ \\
\hline $\operatorname{Pr} 1^{\mathrm{xii}}-\operatorname{Pr} 2-\operatorname{Pr} 3^{\mathrm{x}}$ & $117.969(7)$ \\
\hline $\operatorname{Pr} 1^{x v}-\operatorname{Pr} 2-\operatorname{Pr} 3^{x i}$ & $149.920(8)$ \\
\hline $\operatorname{Pr} 2^{\mathrm{i}}-\operatorname{Pr} 2-\operatorname{Pr} 3^{\mathrm{x}}$ & $112.953(9)$ \\
\hline $\operatorname{Pr} 3^{\mathrm{vii}-}-\operatorname{Pr} 2-\operatorname{Pr} 2^{\mathrm{i}}$ & $59.445(6)$ \\
\hline $\operatorname{Pr} 3^{x i}-\operatorname{Pr} 2-\operatorname{Pr} 2^{i}$ & $60.092(6)$ \\
\hline $\operatorname{Pr} 3^{x i}-\operatorname{Pr} 2-\operatorname{Pr} 3^{x}$ & $61.068(5)$ \\
\hline $\operatorname{Pr} 3^{\mathrm{xi}}-\operatorname{Pr} 2-\operatorname{Pr} 3^{\mathrm{vii}}$ & $65.880(8)$ \\
\hline $\operatorname{Pr} 3^{\mathrm{vii}}-\operatorname{Pr} 2-\operatorname{Pr} 3^{\mathrm{x}}$ & $117.851(7)$ \\
\hline $\mathrm{Si} 1^{\mathrm{xi}}-\operatorname{Pr} 2-\operatorname{Pr} 1^{\mathrm{xv}}$ & $57.51(2)$ \\
\hline 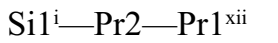 & $96.00(2)$ \\
\hline $\mathrm{Si} 1^{\mathrm{xii}}-\operatorname{Pr} 2-\operatorname{Pr} 1^{\mathrm{xii}}$ & $55.47(2)$ \\
\hline 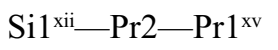 & $57.49(2)$ \\
\hline 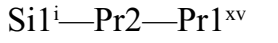 & $55.69(2)$ \\
\hline 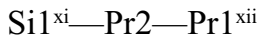 & $147.18(3)$ \\
\hline $\mathrm{Si}^{\mathrm{xii}}-\operatorname{Pr} 2-\operatorname{Pr} 2^{\mathrm{i}}$ & $111.25(2)$ \\
\hline $\mathrm{Si}^{\mathrm{xi}}{ }^{\mathrm{i}} \operatorname{Pr} 2-\operatorname{Pr} 2^{\mathrm{i}}$ & $155.46(3)$ \\
\hline 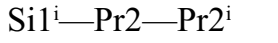 & $90.03(3)$ \\
\hline $\operatorname{Si} 1^{\mathrm{xi}}-\operatorname{Pr} 2-\operatorname{Pr} 3^{\mathrm{vii}}$ & $104.88(3)$ \\
\hline $\mathrm{Si}^{\mathrm{xi}}-\operatorname{Pr} 2-\operatorname{Pr} 3^{\mathrm{x}}$ & $54.93(2)$ \\
\hline Si1 $1^{i}-\operatorname{Pr} 2-\operatorname{Pr} 3^{x}$ & $145.54(2)$ \\
\hline $\mathrm{Si}_{1}{ }^{\mathrm{i}}-\mathrm{Pr} 2-\mathrm{Pr} 3^{\mathrm{vii}}$ & $51.57(2)$ \\
\hline $\mathrm{Si} 1^{\mathrm{xi}}-\operatorname{Pr} 2-\operatorname{Pr} 3^{\mathrm{xi}}$ & $97.03(3)$ \\
\hline Si1 $1^{\mathrm{xii}}-\operatorname{Pr} 2-\operatorname{Pr} 3^{\mathrm{x}}$ & $101.67(2)$ \\
\hline $\mathrm{Si} 1^{\mathrm{xii}}-\operatorname{Pr} 2-\operatorname{Pr} 3^{\mathrm{xi}}$ & $147.77(2)$ \\
\hline $\mathrm{Si}^{\mathrm{i}}-\operatorname{Pr} 2-\operatorname{Pr} 3^{\mathrm{xi}}$ & $117.17(2)$ \\
\hline
\end{tabular}

\begin{tabular}{|c|c|}
\hline $\mathrm{Si} 2-\operatorname{Pr} 3-\operatorname{Pr} 2^{\mathrm{xiii}}$ & $48.31(2)$ \\
\hline Si2-Pr3-Pr2 $2^{\text {xvii }}$ & $103.99(2)$ \\
\hline $\mathrm{Si} 2^{\mathrm{xii}}-\operatorname{Pr} 3-\operatorname{Pr} 2^{\mathrm{xvii}}$ & $110.50(2)$ \\
\hline $\mathrm{Si} 2-\operatorname{Pr} 3-\operatorname{Pr} 2^{x}$ & $103.11(2)$ \\
\hline $\mathrm{Si} 2^{\mathrm{xii}}-\operatorname{Pr} 3-\operatorname{Pr} 2^{\mathrm{x}}$ & $47.59(2)$ \\
\hline $\mathrm{Si} 2^{\mathrm{xiii}}-\operatorname{Pr} 3-\operatorname{Pr} 3^{\mathrm{ii}}$ & $67.57(2)$ \\
\hline $\mathrm{Si} 2-\operatorname{Pr} 3-\operatorname{Pr} 3^{\mathrm{ii}}$ & $50.90(2)$ \\
\hline $\mathrm{Si} 2^{\mathrm{xii}}-\operatorname{Pr} 3-\operatorname{Pr} 3^{\mathrm{ii}}$ & $133.74(2)$ \\
\hline $\mathrm{Si} 2^{\mathrm{xii}}-\mathrm{Pr} 3-\mathrm{Si}^{\mathrm{xiv}}$ & $86.34(3)$ \\
\hline $\mathrm{Si} 2-\mathrm{Pr} 3-\mathrm{Si}^{\mathrm{xii}}$ & $86.35(3)$ \\
\hline $\mathrm{Si} 2^{\mathrm{xiii}}-\operatorname{Pr} 3-\mathrm{Si} 1^{\mathrm{xii}}$ & $175.55(3)$ \\
\hline $\mathrm{Si} 2^{\mathrm{xii}}-\operatorname{Pr} 3-\mathrm{Si}^{\mathrm{xii}}$ & $44.54(3)$ \\
\hline $\mathrm{Si} 2-\operatorname{Pr} 3-\mathrm{Si}^{\mathrm{xiv}}$ & $92.48(3)$ \\
\hline $\mathrm{Si} 2-\mathrm{Pr} 3-\mathrm{Si} 1^{\mathrm{xiii}}$ & $136.40(3)$ \\
\hline $\mathrm{Si} 2-\mathrm{Pr} 3-\mathrm{Si} 2^{\mathrm{xiii}}$ & $91.079(17)$ \\
\hline $\mathrm{Si} 2^{\mathrm{xii}}-\mathrm{Pr} 3-\mathrm{Si} 2^{\mathrm{xiii}}$ & $138.014(18)$ \\
\hline $\mathrm{Si} 2-\mathrm{Pr} 3-\mathrm{Si} 2^{\mathrm{xii}}$ & $130.86(2)$ \\
\hline $\operatorname{Pr} 1-\operatorname{Si} 1-\operatorname{Pr} 1^{\mathrm{xvii}}$ & $123.37(4)$ \\
\hline $\operatorname{Pr} 1-\operatorname{Si} 1-\operatorname{Pr} 2^{i}$ & $68.56(3)$ \\
\hline $\operatorname{Pr} 1-\mathrm{Si} 1-\operatorname{Pr} 3^{\mathrm{ii}}$ & $70.13(2)$ \\
\hline $\operatorname{Pr} 1^{\mathrm{xvii}}-\mathrm{Si} 1-\operatorname{Pr} 3^{\mathrm{ii}}$ & $134.03(4)$ \\
\hline $\operatorname{Pr} 1^{\mathrm{xvii}}-\mathrm{Si} 1-\operatorname{Pr} 3^{\mathrm{v}}$ & $71.14(3)$ \\
\hline $\operatorname{Pr} 1-\operatorname{Si} 1-\operatorname{Pr} 3^{v}$ & $70.70(3)$ \\
\hline $\operatorname{Pr} 1-\operatorname{Si} 1-\operatorname{Pr} 3^{\mathrm{xi}}$ & $136.94(4)$ \\
\hline $\operatorname{Pr} 2^{x i i i}-\operatorname{Si} 1-\operatorname{Pr} 1^{x v i i}$ & $68.02(3)$ \\
\hline $\operatorname{Pr} 2^{\mathrm{ii}}-\mathrm{Si} 1-\operatorname{Pr} 1$ & $69.90(3)$ \\
\hline $\operatorname{Pr} 2^{\text {xiii- }}-\mathrm{Si} 1-\operatorname{Pr} 1$ & $139.92(4)$ \\
\hline $\operatorname{Pr} 2^{\mathrm{ii}}-\mathrm{Si} 1-\operatorname{Pr} 1^{\mathrm{xvii}}$ & $67.99(3)$ \\
\hline $\operatorname{Pr} 2^{i}-\operatorname{Si} 1-\operatorname{Pr} 1^{\mathrm{xvii}}$ & $141.38(4)$ \\
\hline $\operatorname{Pr} 2^{\mathrm{xiii}}-\operatorname{Si} 1-\operatorname{Pr} 2^{\mathrm{i}}$ & $129.66(4)$ \\
\hline $\operatorname{Pr} 2^{\mathrm{ii}}-\mathrm{Si} 1-\operatorname{Pr} 2^{\mathrm{i}}$ & $138.39(4)$ \\
\hline $\operatorname{Pr} 2^{\mathrm{xiii}}-\operatorname{Si} 1-\operatorname{Pr} 2^{\mathrm{ii}}$ & $83.22(3)$ \\
\hline $\operatorname{Pr} 2^{x i i}-\operatorname{Si} 1-\operatorname{Pr} 3^{x i}$ & $82.63(3)$ \\
\hline $\operatorname{Pr} 2^{\text {xiii }}-\operatorname{Si} 1-\operatorname{Pr} 3^{\text {ii }}$ & $76.47(3)$ \\
\hline $\operatorname{Pr} 2^{\mathrm{ii}}-\mathrm{Si} 1-\operatorname{Pr} 3^{\mathrm{xi}}$ & $138.21(4)$ \\
\hline $\operatorname{Pr} 2^{\mathrm{ii}}-\mathrm{Si} 1-\operatorname{Pr} 3^{\mathrm{v}}$ & $87.20(3)$ \\
\hline $\operatorname{Pr} 2^{\mathrm{ii}}-\mathrm{Si} 1-\operatorname{Pr} 3^{\mathrm{ii}}$ & $79.97(3)$ \\
\hline $\operatorname{Pr} 2^{i}-\operatorname{Si} 1-\operatorname{Pr} 3^{x i}$ & $78.10(3)$ \\
\hline $\operatorname{Pr} 2^{\mathrm{i}}-\mathrm{Si} 1-\operatorname{Pr} 3^{\mathrm{ii}}$ & $84.10(3)$ \\
\hline $\operatorname{Pr} 2^{\mathrm{i}}-\mathrm{Si} 1-\operatorname{Pr} 3^{\mathrm{v}}$ & $81.41(3)$ \\
\hline $\operatorname{Pr} 2^{\text {xiii }}-\operatorname{Si} 1-\operatorname{Pr} 3^{v}$ & $138.72(4)$ \\
\hline $\operatorname{Pr} 3^{x i}-\operatorname{Si} 1-\operatorname{Pr} 1^{x v i i}$ & $70.24(3)$ \\
\hline $\operatorname{Pr} 3^{x i}-\operatorname{Si} 1-\operatorname{Pr} 3^{v}$ & $78.24(3)$ \\
\hline $\operatorname{Pr} 3^{x i}-\mathrm{Si} 1-\operatorname{Pr} 3^{\mathrm{ii}}$ & $133.50(4)$ \\
\hline $\operatorname{Pr} 3^{\mathrm{v}}-\mathrm{Si} 1-\operatorname{Pr} 3^{\mathrm{ii}}$ & $140.82(4)$ \\
\hline $\mathrm{Si} 2-\mathrm{Si} 1-\mathrm{Pr} 1$ & $116.82(5)$ \\
\hline $\mathrm{Si} 2-\mathrm{Si} 1-\operatorname{Pr} 1^{\mathrm{xvii}}$ & $119.66(5)$ \\
\hline Si2-Si1-Pr2 ${ }^{\mathrm{xiii}}$ & $63.92(4)$ \\
\hline
\end{tabular}




\begin{tabular}{|c|c|c|c|}
\hline $\mathrm{Si}^{\mathrm{xii}}-\operatorname{Pr} 2-\operatorname{Pr} 3^{\mathrm{vii}}$ & $140.10(2)$ & $\mathrm{Si} 2-\mathrm{Si} 1-\operatorname{Pr} 2^{\mathrm{ii}}$ & $135.25(5)$ \\
\hline $\mathrm{Si} 1^{\mathrm{xii}}-\operatorname{Pr} 2-\mathrm{Si}^{\mathrm{i}}$ & $92.601(18)$ & $\mathrm{Si} 2-\mathrm{Si} 1-\operatorname{Pr} 2^{\mathrm{i}}$ & $65.79(4)$ \\
\hline $\mathrm{Si} 1^{\mathrm{xi}}-\mathrm{Pr} 2-\mathrm{Si}^{\mathrm{i}}$ & $93.56(3)$ & $\mathrm{Si} 2-\mathrm{Si} 1-\operatorname{Pr} 3^{\mathrm{v}}$ & $137.54(6)$ \\
\hline $\mathrm{Si} 1^{\mathrm{xi}}-\mathrm{Pr} 2-\mathrm{Si}^{\mathrm{xii}}$ & $92.85(4)$ & $\mathrm{Si} 2-\mathrm{Si} 1-\operatorname{Pr} 3^{\mathrm{ii}}$ & $64.03(4)$ \\
\hline $\mathrm{Si} 1^{\mathrm{xi}}-\mathrm{Pr} 2-\mathrm{Si} 2^{\mathrm{i}}$ & $140.79(3)$ & $\mathrm{Si} 2-\mathrm{Si} 1-\operatorname{Pr} 3^{\mathrm{xi}}$ & $69.49(4)$ \\
\hline $\mathrm{Si} 1^{\mathrm{xii}}-\mathrm{Pr} 2-\mathrm{Si}^{2}{ }^{\mathrm{i}}$ & $90.38(3)$ & $\operatorname{Pr} 1^{x i i}-\operatorname{Si} 2-\operatorname{Pr} 3^{x i}$ & $135.69(4)$ \\
\hline 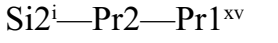 & $92.68(2)$ & $\operatorname{Pr} 2-\mathrm{Si} 2-\operatorname{Pr} 1^{\mathrm{xii}}$ & $70.30(3)$ \\
\hline $\operatorname{Si} 2-\operatorname{Pr} 2-\operatorname{Pr} 1^{x i i}$ & $57.90(2)$ & $\operatorname{Pr} 2^{\mathrm{xii}}-\mathrm{Si} 2-\operatorname{Pr} 1^{\mathrm{xii}}$ & $141.97(4)$ \\
\hline $\operatorname{Si} 2^{x i}-\operatorname{Pr} 2-\operatorname{Pr} 1^{x i i}$ & $158.04(2)$ & $\operatorname{Pr} 2^{\mathrm{i}}-\mathrm{Si} 2-\operatorname{Pr} 1^{\mathrm{xii}}$ & $68.78(3)$ \\
\hline $\mathrm{Si} 2^{\mathrm{i}}-\operatorname{Pr} 2-\operatorname{Pr} 1^{\mathrm{xii}}$ & $57.02(2)$ & $\operatorname{Pr} 2-\mathrm{Si} 2-\operatorname{Pr} 2^{\text {xiii }}$ & $132.15(4)$ \\
\hline $\operatorname{Si} 2-\operatorname{Pr} 2-\operatorname{Pr} 1^{x v}$ & $152.08(2)$ & $\operatorname{Pr} 2-\mathrm{Si} 2-\operatorname{Pr} 2^{\mathrm{i}}$ & $82.12(3)$ \\
\hline $\operatorname{Si} 2^{x i}-\operatorname{Pr} 2-\operatorname{Pr} 1^{x v}$ & $98.16(2)$ & $\operatorname{Pr} 2^{x i i i}-\mathrm{Si} 2-\operatorname{Pr} 2^{\mathrm{i}}$ & $134.64(4)$ \\
\hline $\mathrm{Si} 2^{\mathrm{xi}}-\operatorname{Pr} 2-\operatorname{Pr} 2^{\mathrm{i}}$ & $107.11(2)$ & $\operatorname{Pr} 2^{\mathrm{xiii}}-\mathrm{Si} 2-\operatorname{Pr} 3^{\mathrm{ii}}$ & $80.47(3)$ \\
\hline $\mathrm{Si} 2-\operatorname{Pr} 2-\operatorname{Pr} 2^{\mathrm{i}}$ & $50.30(2)$ & $\operatorname{Pr} 2^{x i i i}-\mathrm{Si} 2-\operatorname{Pr} 3^{x i}$ & $82.34(3)$ \\
\hline $\mathrm{Si} 2^{\mathrm{i}}-\operatorname{Pr} 2-\operatorname{Pr} 2^{\mathrm{i}}$ & $47.57(2)$ & $\operatorname{Pr} 2-\mathrm{Si} 2-\operatorname{Pr} 3^{\mathrm{ii}}$ & $140.09(4)$ \\
\hline $\mathrm{Si} 2^{\mathrm{i}}-\operatorname{Pr} 2-\operatorname{Pr} 3^{\mathrm{vii}}$ & $53.61(2)$ & $\operatorname{Pr} 2^{\mathrm{i}}-\mathrm{Si} 2-\operatorname{Pr} 3^{\mathrm{ii}}$ & $87.54(3)$ \\
\hline $\mathrm{Si} 2^{\mathrm{xi}}-\operatorname{Pr} 2-\operatorname{Pr} 3^{\mathrm{x}}$ & $51.94(2)$ & $\operatorname{Pr} 2-\mathrm{Si} 2-\operatorname{Pr} 3^{\mathrm{xi}}$ & $78.08(3)$ \\
\hline $\mathrm{Si} 2^{\mathrm{xi}}-\operatorname{Pr} 2-\operatorname{Pr} 3^{\mathrm{xi}}$ & $52.15(2)$ & $\operatorname{Pr} 2-\mathrm{Si} 2-\operatorname{Pr} 3^{\mathrm{xi}}$ & $76.97(3)$ \\
\hline $\mathrm{Si} 2^{\mathrm{xi}}-\operatorname{Pr} 2-\operatorname{Pr} 3^{\mathrm{vii}}$ & $70.89(2)$ & $\operatorname{Pr} 2^{\text {xiii- }}-\mathrm{Si} 2-\operatorname{Pr} 3$ & $79.53(3)$ \\
\hline $\mathrm{Si} 2^{\mathrm{i}}-\operatorname{Pr} 2-\operatorname{Pr} 3^{\mathrm{xi}}$ & $100.75(2)$ & $\operatorname{Pr} 2-\mathrm{Si} 2-\operatorname{Pr} 3$ & $85.15(3)$ \\
\hline $\operatorname{Si} 2-\operatorname{Pr} 2-\operatorname{Pr} 3^{x i}$ & $54.47(2)$ & $\operatorname{Pr} 2-\mathrm{Si} 2-\operatorname{Pr} 3$ & $140.56(4)$ \\
\hline $\mathrm{Si} 2^{\mathrm{i}}-\operatorname{Pr} 2-\operatorname{Pr} 3^{\mathrm{x}}$ & $160.33(3)$ & $\operatorname{Pr} 3-\mathrm{Si} 2-\operatorname{Pr} 1^{\mathrm{xii}}$ & $71.78(3)$ \\
\hline $\mathrm{Si} 2-\operatorname{Pr} 2-\operatorname{Pr} 3^{\mathrm{x}}$ & $70.64(3)$ & $\operatorname{Pr} 3^{3 i}-S i 2-\operatorname{Pr} 1^{x i i}$ & $70.02(3)$ \\
\hline $\mathrm{Si} 2-\operatorname{Pr} 2-\operatorname{Pr} 3^{\text {vii }}$ & $102.52(2)$ & $\operatorname{Pr} 3-\mathrm{Si} 2-\operatorname{Pr} 3^{\mathrm{xi}}$ & $136.07(4)$ \\
\hline $\mathrm{Si} 2-\mathrm{Pr} 2-\mathrm{Si}^{\mathrm{xii}}$ & $95.16(3)$ & $\operatorname{Pr} 3-\mathrm{Si} 2-\operatorname{Pr} 3^{\mathrm{ii}}$ & $78.78(3)$ \\
\hline $\mathrm{Si} 2-\mathrm{Pr} 2-\mathrm{Si}^{\mathrm{i}}$ & $139.57(4)$ & $\operatorname{Pr} 3^{3 i}-\mathrm{Si} 2-\operatorname{Pr} 3^{x i}$ & $136.58(4)$ \\
\hline $\mathrm{Si} 2^{\mathrm{i}}-\mathrm{Pr} 2-\mathrm{Si}^{\mathrm{i}}$ & $47.24(3)$ & Si 1 -Si2—Pr $1^{x i i}$ & $121.26(5)$ \\
\hline $\mathrm{Si} 2^{\mathrm{xi}}-\mathrm{Pr} 2-\mathrm{Si}^{\mathrm{xii}}$ & $140.11(3)$ & $\mathrm{Si} 1-\mathrm{Si} 2-\mathrm{Pr} 2^{\mathrm{i}}$ & $66.96(4)$ \\
\hline $\mathrm{Si} 2^{\mathrm{xi}}-\mathrm{Pr} 2-\mathrm{Si}^{\mathrm{i}}$ & $97.97(3)$ & $\mathrm{Si} 1-\mathrm{Si} 2-\mathrm{Pr} 2^{\mathrm{xiii}}$ & $67.74(4)$ \\
\hline $\mathrm{Si} 2^{\mathrm{xi}}-\mathrm{Pr} 2-\mathrm{Si}^{\mathrm{xi}}$ & $48.35(3)$ & $\mathrm{Si} 1-\mathrm{Si} 2-\mathrm{Pr} 2$ & $135.86(5)$ \\
\hline $\mathrm{Si} 2-\mathrm{Pr} 2-\mathrm{Si} 1^{\mathrm{xi}}$ & $125.48(3)$ & $\mathrm{Si} 1-\mathrm{Si} 2-\operatorname{Pr} 3^{\mathrm{ii}}$ & $71.43(4)$ \\
\hline $\mathrm{Si} 2-\mathrm{Pr} 2-\mathrm{Si} 2^{\mathrm{xi}}$ & $100.995(18)$ & $\mathrm{Si} 1-\mathrm{Si} 2-\mathrm{Pr} 3$ & $138.49(5)$ \\
\hline $\mathrm{Si} 2-\mathrm{Pr} 2-\mathrm{Si} 2^{\mathrm{i}}$ & $93.03(4)$ & $\mathrm{Si} 1-\mathrm{Si} 2-\operatorname{Pr} 3^{\mathrm{xi}}$ & $65.16(4)$ \\
\hline
\end{tabular}

Symmetry codes: (i) $-y+1,-x+1,-z+1 / 2$; (ii) $-y+3 / 2, x+1 / 2, z+1 / 4$; (iii) $x+1 / 2,-y+3 / 2,-z+3 / 4$; (iv) $-x+1,-y+1, z+1 / 2$; (v) $-y+2,-x+1,-z+1 / 2$; (vi) $-x+1$, $-y+2, z+1 / 2$; (vii) $-y+3 / 2, x-1 / 2, z+1 / 4$; (viii) $y, x,-z+1$; (ix) $x-1 / 2,-y+3 / 2,-z+3 / 4$; (x) $y, x,-z$; (xi) $-x+3 / 2, y-1 / 2,-z+1 / 4$; (xii) $y-1 / 2,-x+3 / 2, z-1 / 4$; (xiii) $-x+3 / 2, y+1 / 2,-z+1 / 4$; (xiv) $-y+1,-x+2,-z+1 / 2$; (xv) $-x+1,-y+1, z-1 / 2$; (xvi) $-x+1,-y+2, z-1 / 2$; (xvii) $y+1 / 2,-x+3 / 2, z-1 / 4$.

Pentaneodymium tetrasiliside (B)

Crystal data

$\mathrm{Nd}_{5} \mathrm{Si}_{4}$

$M_{r}=833.56$

Tetragonal, $P 4{ }_{1} 2{ }_{1} 2$

Hall symbol: P 4abw 2nw

$a=7.8644$ (2) $\AA$

$c=14.8085(5) \AA$

$V=915.89(6) \AA^{3}$

$Z=4$

$F(000)=1424$
$D_{\mathrm{x}}=6.045 \mathrm{Mg} \mathrm{m}^{-3}$

Mo $K \alpha$ radiation, $\lambda=0.71073 \AA$

Cell parameters from 3985 reflections

$\theta=3.8-30.4^{\circ}$

$\mu=28.27 \mathrm{~mm}^{-1}$

$T=223 \mathrm{~K}$

Plate, metallic gray

$0.12 \times 0.09 \times 0.07 \mathrm{~mm}$ 


\section{Data collection}

XtaLAB AFC12 (RINC): Kappa dual offset/far diffractometer

Radiation source: micro-focus sealed X-ray tube Mirror monochromator

Detector resolution: 5.8140 pixels $\mathrm{mm}^{-1}$

$\omega$ scans

Absorption correction: analytical

[CrysAlisPro (Rigaku OD, 2019) based on

Clark \& Reid (1995)]

Refinement

Refinement on $F^{2}$

Least-squares matrix: full

$R\left[F^{2}>2 \sigma\left(F^{2}\right)\right]=0.015$

$w R\left(F^{2}\right)=0.028$

$S=1.09$

1238 reflections

43 parameters

0 restraints

Primary atom site location: dual

$w=1 /\left[\sigma^{2}\left(F_{\mathrm{o}}^{2}\right)+0.4858 P\right]$

where $P=\left(F_{\mathrm{o}}{ }^{2}+2 F_{\mathrm{c}}{ }^{2}\right) / 3$
$T_{\min }=0.561, T_{\max }=0.702$

6054 measured reflections

1238 independent reflections

1203 reflections with $I>2 \sigma(I)$

$R_{\text {int }}=0.032$

$\theta_{\max }=30.2^{\circ}, \theta_{\min }=3.7^{\circ}$

$h=-11 \rightarrow 10$

$k=-10 \rightarrow 10$

$l=-20 \rightarrow 19$

$(\Delta / \sigma)_{\max }=0.001$

$\Delta \rho_{\max }=1.10 \mathrm{e} \AA^{-3}$

$\Delta \rho_{\min }=-0.71$ e $\AA^{-3}$

Extinction correction: SHELXL (Sheldrick, $2015 \mathrm{~b}), \mathrm{Fc}^{*}=\mathrm{kFc}\left[1+0.001 \mathrm{xFc}^{2} \lambda^{3} / \sin (2 \theta)\right]^{-1 / 4}$

Extinction coefficient: 0.00090 (6)

Absolute structure: Flack $x$ determined using

422 quotients $\left[\left(I^{+}\right)-\left(I^{-}\right)\right] /\left[\left(I^{+}\right)+\left(I^{-}\right)\right]$(Parsons et al., 2013)

Absolute structure parameter: $-0.01(3)$

\section{Special details}

Geometry. All esds (except the esd in the dihedral angle between two 1.s. planes) are estimated using the full covariance matrix. The cell esds are taken into account individually in the estimation of esds in distances, angles and torsion angles; correlations between esds in cell parameters are only used when they are defined by crystal symmetry. An approximate (isotropic) treatment of cell esds is used for estimating esds involving l.s. planes.

Fractional atomic coordinates and isotropic or equivalent isotropic displacement parameters $\left(\hat{A}^{2}\right)$

\begin{tabular}{lllll}
\hline & $x$ & $y$ & $z$ & $U_{\text {iso }} * / U_{\text {eq }}$ \\
\hline $\mathrm{Nd} 1$ & $0.81184(4)$ & $0.81184(4)$ & 0.500000 & $0.00637(9)$ \\
$\mathrm{Nd} 2$ & $0.51490(3)$ & $0.36933(4)$ & $0.12498(2)$ & $0.00518(7)$ \\
$\mathrm{Nd} 3$ & $0.51034(4)$ & $0.87021(4)$ & $0.04676(2)$ & $0.00649(7)$ \\
$\mathrm{Si} 1$ & $0.9274(2)$ & $0.7098(2)$ & $0.30921(10)$ & $0.0060(3)$ \\
$\mathrm{Si} 2$ & $0.7003(2)$ & $0.6636(2)$ & $0.19543(10)$ & $0.0078(3)$ \\
\hline
\end{tabular}

Atomic displacement parameters $\left(\AA^{2}\right)$

\begin{tabular}{lllllll}
\hline & $U^{11}$ & $U^{22}$ & $U^{33}$ & $U^{12}$ & $U^{13}$ & $U^{23}$ \\
\hline $\mathrm{Nd} 1$ & $0.00630(11)$ & $0.00630(11)$ & $0.00652(17)$ & $-0.00026(13)$ & $0.00064(11)$ & $-0.00064(11)$ \\
$\mathrm{Nd} 2$ & $0.00538(14)$ & $0.00534(14)$ & $0.00483(12)$ & $0.00002(12)$ & $0.00035(11)$ & $-0.00097(10)$ \\
$\mathrm{Nd} 3$ & $0.00617(15)$ & $0.00631(14)$ & $0.00700(13)$ & $-0.00027(9)$ & $-0.00098(11)$ & $0.00152(11)$ \\
$\mathrm{Si} 1$ & $0.0060(7)$ & $0.0066(7)$ & $0.0054(6)$ & $-0.0007(5)$ & $0.0001(5)$ & $0.0001(6)$ \\
$\mathrm{Si} 2$ & $0.0069(7)$ & $0.0073(7)$ & $0.0093(6)$ & $-0.0016(5)$ & $-0.0015(6)$ & $0.0017(6)$ \\
\hline
\end{tabular}

Geometric parameters $\left(\AA,{ }^{\circ}\right)$

\begin{tabular}{llll}
\hline $\mathrm{Nd} 1-\mathrm{Nd} 2^{\mathrm{i}}$ & $3.4725(5)$ & $\mathrm{Nd} 2-\mathrm{Nd} 3^{\text {vii }}$ & $3.9061(4)$ \\
$\mathrm{Nd} 1-\mathrm{Nd} 2^{\mathrm{ii}}$ & $3.5021(5)$ & $\mathrm{Nd} 2-\mathrm{Si}^{\mathrm{x}}$ & $3.0366(16)$
\end{tabular}




\begin{tabular}{|c|c|c|c|}
\hline $\mathrm{Nd} 1-\mathrm{Nd} 2^{\mathrm{iii}}$ & $3.5021(5)$ & $\mathrm{Nd} 2-\mathrm{Si}^{\mathrm{i}}$ & $3.0848(17)$ \\
\hline $\mathrm{Nd} 1-\mathrm{Nd} 2^{\mathrm{iv}}$ & $3.4725(5)$ & $\mathrm{Nd} 2-\mathrm{Si}^{\mathrm{xii}}$ & $3.0436(17)$ \\
\hline $\mathrm{Nd} 1-\mathrm{Nd} 3^{\mathrm{v}}$ & $3.6265(3)$ & $\mathrm{Nd} 2-\mathrm{Si} 2$ & $2.9272(17)$ \\
\hline $\mathrm{Nd} 1-\mathrm{Nd} 3^{\mathrm{vi}}$ & $3.6265(3)$ & $\mathrm{Nd} 2-\mathrm{Si}^{\mathrm{x}}$ & $2.9533(17)$ \\
\hline $\mathrm{Nd} 1-\mathrm{Si}^{\mathrm{vii}}$ & $3.1528(16)$ & $\mathrm{Nd} 2-\mathrm{Si} 2^{\mathrm{i}}$ & $3.0565(15)$ \\
\hline $\mathrm{Nd} 1-\mathrm{Si}_{1}{ }^{\mathrm{viii}}$ & $3.0744(15)$ & $\mathrm{Nd} 3-\mathrm{Nd} 3^{\mathrm{xii}}$ & $3.9752(2)$ \\
\hline $\mathrm{Nd} 1-\mathrm{Si} 1$ & $3.0744(15)$ & $\mathrm{Nd} 3-\mathrm{Si}^{\mathrm{xiii}}$ & $3.1359(15)$ \\
\hline $\mathrm{Nd} 1-\mathrm{Si} 1^{\mathrm{ix}}$ & $3.1528(16)$ & $\mathrm{Nd} 3-\mathrm{Si}^{\mathrm{xii}}$ & $3.3315(14)$ \\
\hline $\mathrm{Nd} 1-\mathrm{Si} 2^{\mathrm{ii}}$ & $3.1661(16)$ & $\mathrm{Nd} 3-\mathrm{Si}^{\mathrm{xiv}}$ & $3.1748(15)$ \\
\hline $\mathrm{Nd} 1-\mathrm{Si} 2^{\mathrm{iii}}$ & $3.1661(16)$ & $\mathrm{Nd} 3-\mathrm{Si}_{2}{ }^{\mathrm{xiii}}$ & $3.2425(15)$ \\
\hline $\mathrm{Nd} 2-\mathrm{Nd} 2^{\mathrm{i}}$ & $3.9202(7)$ & $\mathrm{Nd} 3-\mathrm{Si}^{2 \mathrm{xi}}$ & $3.1619(16)$ \\
\hline $\mathrm{Nd} 2-\mathrm{Nd} 3^{x}$ & $3.9094(4)$ & $\mathrm{Nd} 3-\mathrm{Si} 2$ & $3.1177(14)$ \\
\hline $\mathrm{Nd} 2-\mathrm{Nd} 3^{\mathrm{xi}}$ & $3.9378(4)$ & $\mathrm{Si} 1-\mathrm{Si} 2$ & $2.482(2)$ \\
\hline $\mathrm{Nd} 2^{\mathrm{i}}-\mathrm{Nd} 1-\mathrm{Nd} 2^{\mathrm{iv}}$ & $71.144(14)$ & $\mathrm{Si} 2^{\mathrm{x}}-\mathrm{Nd} 2-\mathrm{Si}^{2}$ & $123.86(5)$ \\
\hline 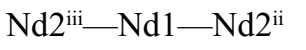 & $68.070(13)$ & $\mathrm{Nd} 1^{\mathrm{xvi}}-\mathrm{Nd} 3-\mathrm{Nd} 1^{\mathrm{xii}}$ & $97.355(7)$ \\
\hline $\mathrm{Nd} 2^{\mathrm{iv}}-\mathrm{Nd} 1-\mathrm{Nd} 2^{\mathrm{ii}}$ & $177.032(7)$ & $\mathrm{Nd} 1^{\mathrm{xvi}}-\mathrm{Nd} 3-\mathrm{Nd} 2^{\mathrm{xi}}$ & $99.740(9)$ \\
\hline $\mathrm{Nd} 2^{\mathrm{i}}-\mathrm{Nd} 1-\mathrm{Nd} 2^{\mathrm{iii}}$ & $177.032(7)$ & $\mathrm{Nd} 1^{\mathrm{xii}}-\mathrm{Nd} 3-\mathrm{Nd} 2^{\mathrm{xiii}}$ & $99.322(9)$ \\
\hline $\mathrm{Nd} 22^{\mathrm{i}}-\mathrm{Nd} 1-\mathrm{Nd} 2^{\mathrm{ii}}$ & $110.453(5)$ & $\mathrm{Nd} 1^{\mathrm{xvi}}-\mathrm{Nd} 3-\mathrm{Nd} 2^{\mathrm{xvii}}$ & $102.777(9)$ \\
\hline $\mathrm{Nd} 2^{\mathrm{iv}}-\mathrm{Nd} 1-\mathrm{Nd} 2^{\mathrm{iii}}$ & $110.453(5)$ & $\mathrm{Nd} 11^{\mathrm{xii}}-\mathrm{Nd} 3-\mathrm{Nd} 2^{\mathrm{xi}}$ & $97.621(8)$ \\
\hline $\mathrm{Nd} 2^{\mathrm{iv}}-\mathrm{Nd} 1-\mathrm{Nd} 3^{\mathrm{v}}$ & $70.374(8)$ & $\mathrm{Nd} 1^{\mathrm{xii}}-\mathrm{Nd} 3-\mathrm{Nd} 2^{\mathrm{xvii}}$ & $158.298(11)$ \\
\hline 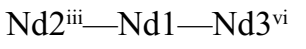 & $106.665(10)$ & $\mathrm{Nd} 1^{\mathrm{xvi}}-\mathrm{Nd} 3-\mathrm{Nd} 2^{\text {xiii }}$ & $136.478(12)$ \\
\hline $\mathrm{Nd} 2^{\mathrm{i}}-\mathrm{Nd} 1-\mathrm{Nd} 3^{\mathrm{v}}$ & $108.985(11)$ & $\mathrm{Nd} 1^{\mathrm{xvi}}-\mathrm{Nd} 3-\mathrm{Nd} 3^{\mathrm{xii}}$ & $57.694(6)$ \\
\hline $\mathrm{Nd} 2^{\mathrm{ii}}-\mathrm{Nd} 1-\mathrm{Nd} 3^{\mathrm{v}}$ & $106.665(10)$ & $\mathrm{Nd} 1^{\mathrm{xii}}-\mathrm{Nd} 3-\mathrm{Nd} 3^{\mathrm{xii}}$ & $137.385(10)$ \\
\hline $\mathrm{Nd} 2^{\mathrm{ii}}-\mathrm{Nd} 1-\mathrm{Nd} 3^{\mathrm{vi}}$ & $73.978(8)$ & $\mathrm{Nd} 2^{\text {xvii }}-\mathrm{Nd} 3-\mathrm{Nd} 2^{\text {xiii }}$ & $60.212(10)$ \\
\hline $\mathrm{Nd} 2^{\mathrm{i}}-\mathrm{Nd} 1-\mathrm{Nd} 3^{\mathrm{vi}}$ & $70.374(8)$ & $\mathrm{Nd} 2^{\mathrm{xii}}-\mathrm{Nd} 3-\mathrm{Nd} 2^{\mathrm{xi}}$ & $117.333(8)$ \\
\hline $\mathrm{Nd} 2^{\mathrm{iv}}-\mathrm{Nd} 1-\mathrm{Nd} 3^{\mathrm{vi}}$ & $108.985(11)$ & $\mathrm{Nd} 2^{\mathrm{xvii}}-\mathrm{Nd} 3-\mathrm{Nd} 2^{\mathrm{xi}}$ & $86.968(8)$ \\
\hline 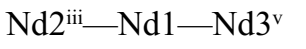 & $73.978(8)$ & $\mathrm{Nd} 2^{\mathrm{xi}}-\mathrm{Nd} 3-\mathrm{Nd} 3^{\mathrm{xii}}$ & $59.212(7)$ \\
\hline $\mathrm{Nd} 3^{\mathrm{vi}}-\mathrm{Nd} 1-\mathrm{Nd} 3^{\mathrm{v}}$ & $179.256(17)$ & $\mathrm{Nd} 2^{\mathrm{xiii}}-\mathrm{Nd} 3-\mathrm{Nd} 3^{\mathrm{xii}}$ & $122.770(9)$ \\
\hline $\mathrm{Si} 1-\mathrm{Nd} 1-\mathrm{Nd} 2^{\mathrm{iii}}$ & $122.55(4)$ & $\mathrm{Nd} 2^{\mathrm{xvii}}-\mathrm{Nd} 3-\mathrm{Nd} 3^{\mathrm{xii}}$ & $62.562(7)$ \\
\hline $\mathrm{Si} 1^{\mathrm{vii}}-\mathrm{Nd} 1-\mathrm{Nd} 2^{\mathrm{i}}$ & $126.97(4)$ & $\mathrm{Si} 1^{\mathrm{xii}}-\mathrm{Nd} 3-\mathrm{Nd} 1^{\mathrm{xvi}}$ & $89.95(3)$ \\
\hline $\mathrm{Si} 1 \mathrm{CNd} 1-\mathrm{Nd} 2^{\mathrm{ii}}$ & $54.67(3)$ & $\mathrm{Si} 1^{\mathrm{xiv}}-\mathrm{Nd} 3-\mathrm{Nd} 1^{\mathrm{xii}}$ & $54.16(3)$ \\
\hline $\mathrm{Si} 1^{\mathrm{ix}}-\mathrm{Nd} 1-\mathrm{Nd} 2^{\mathrm{iii}}$ & $128.53(3)$ & $\mathrm{Si} 1^{\mathrm{xiii}}-\mathrm{Nd} 3-\mathrm{Nd} 1^{\mathrm{xvi}}$ & $55.00(3)$ \\
\hline $\mathrm{Si} 1^{\mathrm{ix}}-\mathrm{Nd} 1-\mathrm{Nd} 2^{\mathrm{iv}}$ & $54.30(3)$ & $\mathrm{Si} 1^{\mathrm{xii}}-\mathrm{Nd} 3-\mathrm{Nd} 1^{\mathrm{xii}}$ & $51.72(3)$ \\
\hline $\mathrm{Si} 1-\mathrm{Nd} 1-\mathrm{Nd} 2^{\mathrm{i}}$ & $55.82(3)$ & $\mathrm{Si} 1^{\mathrm{xii}}-\mathrm{Nd} 3-\mathrm{Nd} 1^{\mathrm{xii}}$ & $143.78(3)$ \\
\hline $\mathrm{Si} 1^{\mathrm{vii}}-\mathrm{Nd} 1-\mathrm{Nd} 2^{\mathrm{iv}}$ & $54.44(3)$ & $\mathrm{Si} 1^{\mathrm{xiv}}-\mathrm{Nd} 3-\mathrm{Nd} 1^{\mathrm{xvi}}$ & $53.25(3)$ \\
\hline $\mathrm{Si} 1^{\mathrm{viii}}-\mathrm{Nd} 1-\mathrm{Nd} 2^{\mathrm{iii}}$ & $54.67(3)$ & $\mathrm{Si} 1^{\mathrm{xii}}-\mathrm{Nd} 3-\mathrm{Nd} 2^{\mathrm{xvii}}$ & $50.52(3)$ \\
\hline $\mathrm{Si} 1^{\mathrm{ix}}-\mathrm{Nd} 1-\mathrm{Nd} 2^{\mathrm{i}}$ & $54.44(3)$ & $\mathrm{Si} 1^{\text {xiv }}-\mathrm{Nd} 3-\mathrm{Nd} 2^{\text {xvii }}$ & $135.69(3)$ \\
\hline 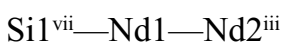 & $124.35(3)$ & $\mathrm{Si} 1^{\mathrm{xii}}-\mathrm{Nd} 3-\mathrm{Nd} 2^{\mathrm{xiii}}$ & $131.30(3)$ \\
\hline $\mathrm{Si} 1^{\mathrm{vii}}-\mathrm{Nd} 1-\mathrm{Nd} 2^{\mathrm{i}}$ & $54.30(3)$ & $\mathrm{Si} 1^{\mathrm{xiv}}-\mathrm{Nd} 3-\mathrm{Nd} 2^{\mathrm{xi}}$ & $129.50(3)$ \\
\hline $\mathrm{Si} 1-\mathrm{Nd} 1-\mathrm{Nd} 2^{\mathrm{iv}}$ & $126.97(4)$ & $\mathrm{Si} 1^{\mathrm{xii}}-\mathrm{Nd} 3-\mathrm{Nd} 2^{\mathrm{xvii}}$ & $135.35(3)$ \\
\hline $\mathrm{Si} 1^{\mathrm{viii}}-\mathrm{Nd} 1-\mathrm{Nd} 2^{\mathrm{ii}}$ & $122.55(4)$ & $\mathrm{Si} 1^{\mathrm{xiii}}-\mathrm{Nd} 3-\mathrm{Nd} 2^{\mathrm{xiii}}$ & $90.04(3)$ \\
\hline $\mathrm{Si} 1^{\mathrm{viii}}-\mathrm{Nd} 1-\mathrm{Nd} 2^{\mathrm{iv}}$ & $55.82(3)$ & $\mathrm{Si} 1^{\mathrm{xii}}-\mathrm{Nd} 3-\mathrm{Nd} 2^{\mathrm{xi}}$ & $48.50(3)$ \\
\hline $\mathrm{Si} 1^{\mathrm{ix}}-\mathrm{Nd} 1-\mathrm{Nd} 2^{\mathrm{ii}}$ & $124.35(3)$ & $\mathrm{Si} 1^{\mathrm{xii}}-\mathrm{Nd} 3-\mathrm{Nd} 2^{\mathrm{xi}}$ & $108.97(3)$ \\
\hline $\mathrm{Si} 1^{\mathrm{vii}}-\mathrm{Nd} 1-\mathrm{Nd} 2^{\mathrm{ii}}$ & $128.53(3)$ & $\mathrm{Si} 1^{\text {xiv }}-\mathrm{Nd} 3-\mathrm{Nd} 2^{\text {xiii }}$ & $108.81(3)$ \\
\hline $\mathrm{Si} 1-\mathrm{Nd} 1-\mathrm{Nd} 3^{\mathrm{vi}}$ & $55.83(3)$ & $\mathrm{Si} 1^{\mathrm{xiii}}-\mathrm{Nd} 3-\mathrm{Nd} 3^{\text {xii }}$ & $51.39(3)$ \\
\hline $\mathrm{Si} 1^{\mathrm{viii}}-\mathrm{Nd} 1-\mathrm{Nd} 3^{\mathrm{v}}$ & $55.83(3)$ & $\mathrm{Si} 1^{\mathrm{xii}}-\mathrm{Nd} 3-\mathrm{Nd} 3^{\mathrm{xii}}$ & $91.19(3)$ \\
\hline $\mathrm{Si}^{\mathrm{vii}}-\mathrm{Nd} 1-\mathrm{Nd} 3^{\mathrm{v}}$ & $124.78(3)$ & $\mathrm{Si} 1^{\text {xiv }}-\mathrm{Nd} 3-\mathrm{Nd} 3^{\text {xii }}$ & $110.85(3)$ \\
\hline
\end{tabular}




\begin{tabular}{|c|c|}
\hline $\mathrm{Si} 1^{\mathrm{vii}}-\mathrm{Nd} 1-\mathrm{Nd} 3^{\mathrm{vi}}$ & $124.19(3)$ \\
\hline $\mathrm{Si} 1^{\mathrm{ix}}-\mathrm{Nd} 1-\mathrm{Nd} 3^{\mathrm{v}}$ & $54.57(3)$ \\
\hline $\mathrm{Si} 1^{\mathrm{ix}}-\mathrm{Nd} 1-\mathrm{Nd} 3^{\mathrm{vi}}$ & $124.78(3)$ \\
\hline 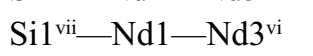 & $54.57(3)$ \\
\hline $\mathrm{Si} 1-\mathrm{Nd} 1-\mathrm{Nd} 3^{\mathrm{v}}$ & $124.19(3)$ \\
\hline Si $1-N d 1-S i 1^{\text {vii }}$ & $91.21(4)$ \\
\hline $\mathrm{Si} 1^{\mathrm{viii}}-\mathrm{Nd} 1-\mathrm{Si} 1^{\mathrm{ix}}$ & $91.21(4)$ \\
\hline $\mathrm{Si} 1-\mathrm{Nd} 1-\mathrm{Si}_{1}{ }^{\mathrm{ix}}$ & $90.79(3)$ \\
\hline $\mathrm{Si} 1^{\mathrm{viii}}-\mathrm{Nd} 1-\mathrm{Si}^{\mathrm{vii}}$ & $90.79(3)$ \\
\hline $\mathrm{Si} 1-\mathrm{Nd} 1-\mathrm{Si} 1^{\mathrm{viii}}$ & $177.21(7)$ \\
\hline $\mathrm{Si} 1^{\mathrm{ix}}-\mathrm{Nd} 1-\mathrm{Si}^{\mathrm{vii}}$ & $88.51(6)$ \\
\hline $\mathrm{Si}^{\mathrm{vii}}-\mathrm{Nd} 1-\mathrm{Si} 2^{\mathrm{ii}}$ & $178.58(4)$ \\
\hline $\mathrm{Si} 1^{\mathrm{ix}}-\mathrm{Nd} 1-\mathrm{Si}_{2}{ }^{\mathrm{ii}}$ & $92.27(4)$ \\
\hline $\mathrm{Si} 1^{\mathrm{vii}}-\mathrm{Nd} 1-\mathrm{Si} 2^{\mathrm{iii}}$ & $92.27(4)$ \\
\hline $\mathrm{Si} 1-\mathrm{Nd} 1-\mathrm{Si} 2^{\mathrm{ii}}$ & $89.96(4)$ \\
\hline $\mathrm{Si} 1^{\mathrm{ix}}-\mathrm{Nd} 1-\mathrm{Si} 2^{\mathrm{iii}}$ & $178.58(4)$ \\
\hline $\mathrm{Si} 1-\mathrm{Nd} 1-\mathrm{Si} 2^{\mathrm{iii}}$ & $88.01(4)$ \\
\hline $\mathrm{Si} 1^{\mathrm{viii}}-\mathrm{Nd} 1-\mathrm{Si} 2^{\mathrm{iii}}$ & $89.96(4)$ \\
\hline 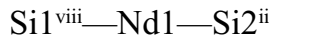 & $88.01(4)$ \\
\hline $\mathrm{Si} 2^{\mathrm{iii}}-\mathrm{Nd} 1-\mathrm{Nd} 2^{\mathrm{ii}}$ & $54.28(3)$ \\
\hline $\mathrm{Si} 2^{\mathrm{iii}}-\mathrm{Nd} 1-\mathrm{Nd} 2^{\mathrm{iv}}$ & $127.09(3)$ \\
\hline $\mathrm{Si} 2^{\mathrm{ii}}-\mathrm{Nd} 1-\mathrm{Nd} 2^{\mathrm{ii}}$ & $51.78(3)$ \\
\hline $\mathrm{Si} 2^{\mathrm{iii}}-\mathrm{Nd} 1-\mathrm{Nd} 2^{\mathrm{i}}$ & $125.25(3)$ \\
\hline 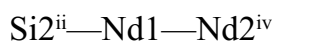 & $125.25(3)$ \\
\hline $\mathrm{Si} 2^{\mathrm{ii}}-\mathrm{Nd} 1-\mathrm{Nd} 2^{\mathrm{iii}}$ & $54.28(3)$ \\
\hline 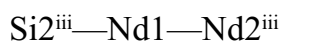 & $51.78(3)$ \\
\hline $\mathrm{Si} 2^{\mathrm{ii}}-\mathrm{Nd} 1-\mathrm{Nd} 2^{\mathrm{i}}$ & $127.09(3)$ \\
\hline $\mathrm{Si} 2^{2 \mathrm{ii}}-\mathrm{Nd} 1-\mathrm{Nd} 3^{\mathrm{vi}}$ & $54.98(3)$ \\
\hline $\mathrm{Si} 2^{\mathrm{ii}}-\mathrm{Nd} 1-\mathrm{Nd} 3^{\mathrm{vi}}$ & 125.68 \\
\hline $\mathrm{Si} 2^{\mathrm{ii}}-\mathrm{Nd} 1-\mathrm{Nd} 3^{\mathrm{v}}$ & $54.98(3)$ \\
\hline 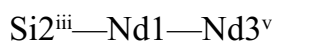 & $125.68(3)$ \\
\hline 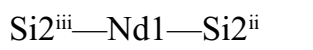 & $86.97(6)$ \\
\hline $\mathrm{Nd} 1^{\mathrm{xv}}-\mathrm{Nd} 2-\mathrm{Nd} 1^{\mathrm{xii}}$ & $103.774(8)$ \\
\hline $\mathrm{Nd} 1^{\mathrm{xi}}-\mathrm{Nd} 2-\mathrm{Nd} 2^{\mathrm{i}}$ & $55.965(6)$ \\
\hline $\mathrm{Nd} 1^{\mathrm{xv}}-\mathrm{Nd} 2-\mathrm{Nd} 2^{\mathrm{i}}$ & $140.405(13)$ \\
\hline $\mathrm{Nd} 1^{\mathrm{xv}}-\mathrm{Nd} 2-\mathrm{Nd} 3^{\mathrm{x}}$ & $149.935(9)$ \\
\hline $\mathrm{Nd} 1^{\mathrm{xv}}-\mathrm{Nd} 2-\mathrm{Nd} 3^{\mathrm{xi}}$ & $107.244(10)$ \\
\hline $\mathrm{Nd} 1^{\mathrm{xii}}-\mathrm{Nd} 2-\mathrm{Nd} 3^{\mathrm{vii}}$ & $106.182(10)$ \\
\hline $\mathrm{Nd} 1^{\mathrm{xii}}-\mathrm{Nd} 2-\mathrm{Nd} 3^{\mathrm{x}}$ & $106.111(9)$ \\
\hline $\mathrm{Nd} 1^{\mathrm{xii}}-\mathrm{Nd} 2-\mathrm{Nd} 3^{\mathrm{xi}}$ & $117.604(9)$ \\
\hline $\mathrm{Nd} 1^{\mathrm{xv}}-\mathrm{Nd} 2-\mathrm{Nd} 3^{\mathrm{vii}}$ & $102.810(9)$ \\
\hline $\mathrm{Nd} 2^{\mathrm{i}}-\mathrm{Nd} 2-\mathrm{Nd} 3^{\mathrm{xi}}$ & $112.321(11)$ \\
\hline $\mathrm{Nd} 3^{\mathrm{x}}-\mathrm{Nd} 2-\mathrm{Nd} 2^{\mathrm{i}}$ & $59.853(7)$ \\
\hline $\mathrm{Nd} 3^{\mathrm{vii}}-\mathrm{Nd} 2-\mathrm{Nd} 2^{\mathrm{i}}$ & $59.937(8)$ \\
\hline $\mathrm{Nd} 3^{\mathrm{vii}}-\mathrm{Nd} 2-\mathrm{Nd} 3^{\mathrm{xi}}$ & $117.388(8)$ \\
\hline $\mathrm{Nd} 3^{\mathrm{vii}}-\mathrm{Nd} 2-\mathrm{Nd} 3^{\mathrm{x}}$ & $65.627(10)$ \\
\hline $\mathrm{Nd} 3^{\mathrm{x}}-\mathrm{Nd} 2-\mathrm{Nd} 3^{\mathrm{xi}}$ & $60.872(7)$ \\
\hline $\mathrm{Si} 1^{\mathrm{x}}-\mathrm{Nd} 2-\mathrm{Nd} 1^{\mathrm{xv}}$ & $57.47(3)$ \\
\hline
\end{tabular}

\begin{tabular}{|c|c|}
\hline $\mathrm{Si} 1^{\mathrm{xiv}}-\mathrm{Nd} 3-\mathrm{Si} 1^{\mathrm{xii}}$ & $85.88(2)$ \\
\hline $\mathrm{Si} 1^{\mathrm{xii}}-\mathrm{Nd} 3-\mathrm{Si}^{\mathrm{xii}}$ & $137.46(2)$ \\
\hline $\mathrm{Si} 1^{\mathrm{xiii}}-\mathrm{Nd} 3-\mathrm{Si}^{\mathrm{xiv}}$ & $89.678(15)$ \\
\hline $\mathrm{Si} 1^{\mathrm{xiv}}-\mathrm{Nd} 3-\mathrm{Si} 2^{\mathrm{xiii}}$ & $90.11(4)$ \\
\hline $\mathrm{Si} 1^{\mathrm{xii}}-\mathrm{Nd} 3-\mathrm{Si} 2^{\mathrm{xiii}}$ & $45.76(4)$ \\
\hline $\mathrm{Si} 1^{\mathrm{xii}}-\mathrm{Nd} 3-\mathrm{Si} 2^{\mathrm{xii}}$ & $92.67(4)$ \\
\hline $\mathrm{Si} 2^{\mathrm{xii}}-\mathrm{Nd} 3-\mathrm{Nd} 1^{\mathrm{xii}}$ & $87.36(3)$ \\
\hline $\mathrm{Si} 2-\mathrm{Nd} 3-\mathrm{Nd} 1^{\mathrm{xii}}$ & $54.76(3)$ \\
\hline $\mathrm{Si} 2-\mathrm{Nd} 3-\mathrm{Nd} 1^{\mathrm{xvi}}$ & $146.00(3)$ \\
\hline $\mathrm{Si} 2^{\mathrm{xiii}}-\mathrm{Nd} 3-\mathrm{Nd} 1^{\mathrm{xii}}$ & $123.04(3)$ \\
\hline 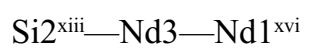 & $90.38(3)$ \\
\hline $\mathrm{Si} 2^{\mathrm{xii}}-\mathrm{Nd} 3-\mathrm{Nd} 1^{\mathrm{xvi}}$ & $55.09(3)$ \\
\hline 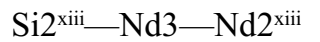 & $47.19(3)$ \\
\hline 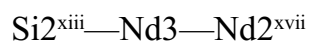 & $49.57(3)$ \\
\hline $\mathrm{Si} 2^{\mathrm{xii}}-\mathrm{Nd} 3-\mathrm{Nd} 2^{\mathrm{xi}}$ & $136.52(3)$ \\
\hline $\mathrm{Si} 2^{\mathrm{xii}}-\mathrm{Nd} 3-\mathrm{Nd} 2^{\mathrm{xvii}}$ & $110.79(3)$ \\
\hline $\mathrm{Si} 2-\mathrm{Nd} 3-\mathrm{Nd} 2^{\mathrm{xvii}}$ & $103.54(3)$ \\
\hline $\mathrm{Si} 2-\mathrm{Nd} 3-\mathrm{Nd} 2^{\text {xiii }}$ & $48.09(3)$ \\
\hline $\mathrm{Si} 2^{\mathrm{xii}}-\mathrm{Nd} 3-\mathrm{Nd} 2^{\mathrm{xiii}}$ & $164.61(3)$ \\
\hline $\mathrm{Si} 2-\mathrm{Nd} 3-\mathrm{Nd} 2^{\mathrm{xi}}$ & $102.77(3)$ \\
\hline $\mathrm{Si} 2^{\mathrm{xii}}-\mathrm{Nd} 3-\mathrm{Nd} 2^{\mathrm{xi}}$ & $47.63(3)$ \\
\hline $\mathrm{Si} 2^{\mathrm{xiii}}-\mathrm{Nd} 3-\mathrm{Nd} 3^{\mathrm{xii}}$ & $93.42(3)$ \\
\hline $\mathrm{Si} 2-\mathrm{Nd} 3-\mathrm{Nd} 3^{\mathrm{xii}}$ & $156.02(3)$ \\
\hline $\mathrm{Si} 2^{\mathrm{xii}}-\mathrm{Nd} 3-\mathrm{Nd} 3^{\mathrm{xii}}$ & $50.23(3)$ \\
\hline $\mathrm{Si} 2^{\mathrm{xii}}-\mathrm{Nd} 3-\mathrm{Si} 1^{\mathrm{xiv}}$ & $86.36(4)$ \\
\hline $\mathrm{Si} 2-\mathrm{Nd} 3-\mathrm{Si}^{\mathrm{xii}}$ & $86.26(4)$ \\
\hline $\mathrm{Si} 2^{\mathrm{xiii}}-\mathrm{Nd} 3-\mathrm{Si} 1^{\mathrm{xii}}$ & $174.74(4)$ \\
\hline $\mathrm{Si} 2^{\mathrm{xii}}-\mathrm{Nd} 3-\mathrm{Si}^{\mathrm{xii}}$ & $44.85(4)$ \\
\hline $\mathrm{Si} 2-\mathrm{Nd} 3-\mathrm{Si}^{\mathrm{xiv}}$ & $92.77(4)$ \\
\hline $\mathrm{Si} 2-\mathrm{Nd} 3-\mathrm{Si} 1^{\mathrm{xiii}}$ & $136.26(4)$ \\
\hline $\mathrm{Si} 2-\mathrm{Nd} 3-\mathrm{Si}^{2^{\mathrm{xiii}}}$ & $90.54(2)$ \\
\hline $\mathrm{Si} 2^{\mathrm{xii}}-\mathrm{Nd} 3-\mathrm{Si} 2^{\mathrm{xiii}}$ & $138.34(2)$ \\
\hline $\mathrm{Si} 2-\mathrm{Nd} 3-\mathrm{Si} 2^{\mathrm{xii}}$ & $131.07(3)$ \\
\hline $\mathrm{Nd} 1-\mathrm{Si} 1-\mathrm{Nd} 1^{\mathrm{xvii}}$ & $123.58(5)$ \\
\hline $\mathrm{Nd} 1-\mathrm{Si} 1-\mathrm{Nd} 2^{\mathrm{i}}$ & $68.64(3)$ \\
\hline $\mathrm{Nd} 1-\mathrm{Si} 1-\mathrm{Nd} 3^{\mathrm{ii}}$ & $70.00(3)$ \\
\hline $\mathrm{Nd} 1^{\mathrm{xvii}}-\mathrm{Si} 1-\mathrm{Nd} 3^{\mathrm{ii}}$ & $134.15(5)$ \\
\hline $\mathrm{Nd} 1^{\mathrm{xvii}}-\mathrm{Si} 1-\mathrm{Nd} 3^{\mathrm{vi}}$ & $71.13(3)$ \\
\hline $\mathrm{Nd} 1-\mathrm{Si} 1-\mathrm{Nd} 3^{\mathrm{vi}}$ & $70.93(3)$ \\
\hline $\mathrm{Nd} 1-\mathrm{Si} 1-\mathrm{Nd}^{\mathrm{x}}$ & $136.75(6)$ \\
\hline $\mathrm{Nd} 2^{\mathrm{xiii}}-\mathrm{Si} 1-\mathrm{Nd} 1^{\mathrm{xvii}}$ & $68.23(3)$ \\
\hline $\mathrm{Nd} 22^{\mathrm{ii}}-\mathrm{Si} 1-\mathrm{Nd} 1$ & $69.84(3)$ \\
\hline $\mathrm{Nd} 2^{\text {xiii }}-\mathrm{Si} 1-\mathrm{Nd} 1$ & $139.62(5)$ \\
\hline $\mathrm{Nd} 2^{\mathrm{ii}}-\mathrm{Si} 1-\mathrm{Nd} 1^{\mathrm{xvii}}$ & $68.14(4)$ \\
\hline $\mathrm{Nd} 2^{\mathrm{i}}-\mathrm{Si} 1-\mathrm{Nd} 1^{\mathrm{xvii}}$ & $141.48(5)$ \\
\hline $\mathrm{Nd} 2^{\mathrm{xiii}}-\mathrm{Si} 1-\mathrm{Nd} 2^{\mathrm{i}}$ & $129.31(5)$ \\
\hline $\mathrm{Nd} 2^{\mathrm{ii}}-\mathrm{Si} 1-\mathrm{Nd} 2^{\mathrm{i}}$ & $138.41(5)$ \\
\hline $\mathrm{Nd} 2^{\mathrm{xii}}-\mathrm{Si} 1-\mathrm{Nd} 2^{\mathrm{ii}}$ & $83.28(4)$ \\
\hline
\end{tabular}




\begin{tabular}{|c|c|}
\hline $\mathrm{Si} 1^{\mathrm{i}}-\mathrm{Nd} 2-\mathrm{Nd} 1^{\mathrm{xii}}$ & $96.51(3)$ \\
\hline 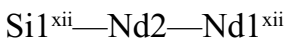 & $55.49(3)$ \\
\hline 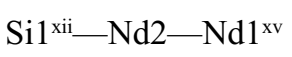 & $57.42(3)$ \\
\hline $\mathrm{Si} 1^{\mathrm{i}}-\mathrm{Nd} 2-\mathrm{Nd} 1^{\mathrm{xv}}$ & $55.54(3)$ \\
\hline $\mathrm{Si}^{\mathrm{x}}-\mathrm{Nd} 2-\mathrm{Nd} 1^{\mathrm{xii}}$ & $147.02(3)$ \\
\hline $\mathrm{Si} 1^{\mathrm{xii}}-\mathrm{Nd} 2-\mathrm{Nd} 2^{\mathrm{i}}$ & $111.31(3)$ \\
\hline $\mathrm{Si} 1^{\mathrm{x}}-\mathrm{Nd} 2-\mathrm{Nd} 2^{\mathrm{i}}$ & $155.46(3)$ \\
\hline $\mathrm{Si} 1^{\mathrm{i}}-\mathrm{Nd} 2-\mathrm{Nd} 2^{\mathrm{i}}$ & $90.59(3)$ \\
\hline $\mathrm{Si} 1^{\mathrm{x}}-\mathrm{Nd} 2-\mathrm{Nd} 3^{\mathrm{x}}$ & $97.14(3)$ \\
\hline $\mathrm{Si}^{\mathrm{x}}-\mathrm{Nd} 2-\mathrm{Nd} 3^{\mathrm{xi}}$ & $55.26(3)$ \\
\hline $\mathrm{Si} 1^{\mathrm{i}}-\mathrm{Nd} 2-\mathrm{Nd} 3^{\mathrm{xi}}$ & $145.44(3)$ \\
\hline 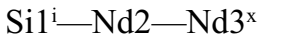 & $117.05(3)$ \\
\hline $\mathrm{Si}^{2}-\mathrm{Nd} 2-\mathrm{Nd} 3^{\mathrm{vii}}$ & $104.58(3)$ \\
\hline $\mathrm{Si}^{\mathrm{xii}}-\mathrm{Nd} 2-\mathrm{Nd} 3^{\mathrm{xi}}$ & $101.82(3)$ \\
\hline 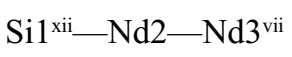 & $140.39(3)$ \\
\hline $\mathrm{Si} 1^{\mathrm{i}}-\mathrm{Nd} 2-\mathrm{Nd} 3^{\mathrm{vii}}$ & $51.69(3)$ \\
\hline $\mathrm{Si}^{\mathrm{xii}}-\mathrm{Nd} 2-\mathrm{Nd} 3^{\mathrm{x}}$ & $147.88(3)$ \\
\hline $\mathrm{Si}^{\mathrm{xii}}-\mathrm{Nd} 2-\mathrm{Si}{ }^{\mathrm{i}}$ & $92.69(2)$ \\
\hline $\mathrm{Si}^{\mathrm{x}}-\mathrm{Nd} 2-\mathrm{Si}^{1}{ }^{\mathrm{i}}$ & $93.26(4)$ \\
\hline $\mathrm{Si}^{\mathrm{x}}-\mathrm{Nd} 2-\mathrm{Si}^{\mathrm{xii}}$ & $92.72(5)$ \\
\hline $\mathrm{Si} 1^{x}-\mathrm{Nd} 2-\mathrm{Si}^{2}$ & $140.91(4)$ \\
\hline $\mathrm{Si} 1^{\mathrm{xii}}-\mathrm{Nd} 2-\mathrm{Si}^{2}{ }^{\mathrm{i}}$ & $90.60(4)$ \\
\hline $\mathrm{Si} 2^{\mathrm{i}}-\mathrm{Nd} 2-\mathrm{Nd} 1^{\mathrm{xv}}$ & $92.89(3)$ \\
\hline $\mathrm{Si} 2-\mathrm{Nd} 2-\mathrm{Nd} 1^{\mathrm{xii}}$ & $58.18(3)$ \\
\hline $\mathrm{Si} 2^{x}-\mathrm{Nd} 2-\mathrm{Nd} 1^{\mathrm{xii}}$ & $157.63(3)$ \\
\hline $\mathrm{Si} 2^{\mathrm{i}}-\mathrm{Nd} 2-\mathrm{Nd} 1^{\mathrm{xii}}$ & $57.24(3)$ \\
\hline $\mathrm{Si} 2-\mathrm{Nd} 2-\mathrm{Nd} 1^{\mathrm{xv}}$ & $151.99(3)$ \\
\hline $\mathrm{Si} 2^{\mathrm{x}}-\mathrm{Nd} 2-\mathrm{Nd} 1^{\mathrm{xv}}$ & $98.52(3)$ \\
\hline $\mathrm{Si} 2^{\mathrm{x}}-\mathrm{Nd} 2-\mathrm{Nd} 2^{\mathrm{i}}$ & 106.53 \\
\hline $\mathrm{Si} 2-\mathrm{Nd} 2-\mathrm{Nd} 2^{\mathrm{i}}$ & $50.51(3)$ \\
\hline $\mathrm{Si} 2^{\mathrm{i}}-\mathrm{Nd} 2-\mathrm{Nd} 2^{\mathrm{i}}$ & $47.66(3)$ \\
\hline 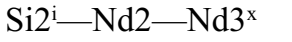 & $100.42(3)$ \\
\hline $\mathrm{Si} 2^{\mathrm{x}}-\mathrm{Nd} 2-\mathrm{Nd} 3^{\mathrm{xi}}$ & $52.28(3)$ \\
\hline $\mathrm{Si}^{2}{ }^{\mathrm{x}}-\mathrm{Nd} 2-\mathrm{Nd} 3^{\mathrm{vii}}$ & $70.03(3)$ \\
\hline $\mathrm{Si} 2^{\mathrm{x}}-\mathrm{Nd} 2-\mathrm{Nd} 3^{\mathrm{x}}$ & $51.78(3)$ \\
\hline $\mathrm{Si} 2^{\mathrm{i}}-\mathrm{Nd} 2-\mathrm{Nd} 3^{\text {vii }}$ & $53.85(3)$ \\
\hline $\mathrm{Si} 2-\mathrm{Nd} 2-\mathrm{Nd} 3^{\mathrm{vii}}$ & $102.94(3)$ \\
\hline $\mathrm{Si} 2^{\mathrm{i}}-\mathrm{Nd} 2-\mathrm{Nd} 3^{\mathrm{xi}}$ & $159.76(3)$ \\
\hline $\mathrm{Si} 2-\mathrm{Nd} 2-\mathrm{Nd} 3^{\mathrm{xi}}$ & $69.76(3)$ \\
\hline $\mathrm{Si} 2-\mathrm{Nd} 2-\mathrm{Nd} 3^{\mathrm{x}}$ & $54.35(3)$ \\
\hline $\mathrm{Si} 2-\mathrm{Nd} 2-\mathrm{Si} 1^{\mathrm{xii}}$ & $95.25(4)$ \\
\hline $\mathrm{Si} 2-\mathrm{Nd} 2-\mathrm{Si}^{\mathrm{i}}{ }^{\mathrm{i}}$ & $140.41(4)$ \\
\hline $\mathrm{Si} 2^{\mathrm{i}}-\mathrm{Nd} 2-\mathrm{Si}^{\mathrm{i}}$ & $47.67(4)$ \\
\hline $\mathrm{Si} 2^{\mathrm{x}}-\mathrm{Nd} 2-\mathrm{Si} 1^{\mathrm{xii}}$ & $140.61(4)$ \\
\hline $\mathrm{Si} 2^{\mathrm{x}}-\mathrm{Nd} 2-\mathrm{Si}^{\mathrm{i}}$ & $97.55(4)$ \\
\hline $\mathrm{Si} 2^{\mathrm{x}}-\mathrm{Nd} 2-\mathrm{Si}{ }^{\mathrm{x}}$ & $48.93(4)$ \\
\hline $\mathrm{Si} 2-\mathrm{Nd} 2-\mathrm{Si}^{\mathrm{x}}$ & $124.91(4)$ \\
\hline
\end{tabular}

\begin{tabular}{|c|c|}
\hline $\mathrm{Nd} 2^{\mathrm{xii}}-\mathrm{Si} 1-\mathrm{Nd} 3^{\mathrm{x}}$ & $83.05(4)$ \\
\hline $\mathrm{Nd} 2^{\mathrm{xii}}-\mathrm{Si} 1-\mathrm{Nd} 3^{\mathrm{ii}}$ & $76.24(3)$ \\
\hline $\mathrm{Nd} 2^{\mathrm{ii}}-\mathrm{Si} 1-\mathrm{Nd} 3^{\mathrm{x}}$ & $138.55(5)$ \\
\hline $\mathrm{Nd} 2^{\mathrm{ii}}-\mathrm{Si} 1-\mathrm{Nd} 3^{\mathrm{vi}}$ & $87.22(4)$ \\
\hline $\mathrm{Nd} 2^{\mathrm{ii}}-\mathrm{Si} 1-\mathrm{Nd} 3^{\mathrm{ii}}$ & $80.06(4)$ \\
\hline $\mathrm{Nd} 2^{\mathrm{i}}-\mathrm{Si} 1-\mathrm{Nd} 3^{\mathrm{x}}$ & $77.79(4)$ \\
\hline $\mathrm{Nd} 2^{\mathrm{i}}-\mathrm{Si} 1-\mathrm{Nd} 3^{\mathrm{ii}}$ & $83.83(4)$ \\
\hline $\mathrm{Nd} 2^{\mathrm{i}}-\mathrm{Si} 1-\mathrm{Nd}^{\mathrm{vi}}$ & $81.65(4)$ \\
\hline $\mathrm{Nd} 2^{\mathrm{xii}}-\mathrm{Si} 1-\mathrm{Nd} 3^{\mathrm{vi}}$ & $138.92(5)$ \\
\hline $\mathrm{Nd} 3^{x}-\mathrm{Si} 1-\mathrm{Nd} 1^{\mathrm{xvii}}$ & $70.43(3)$ \\
\hline $\mathrm{Nd} 3^{\mathrm{x}}-\mathrm{Si} 1-\mathrm{Nd} 3^{\mathrm{vi}}$ & $78.09(3)$ \\
\hline $\mathrm{Nd} 3^{\mathrm{x}}-\mathrm{Si} 1-\mathrm{Nd} 3^{\mathrm{ii}}$ & $133.33(5)$ \\
\hline $\mathrm{Nd} 3^{\mathrm{vi}}-\mathrm{Si} 1-\mathrm{Nd} 3^{\mathrm{ii}}$ & $140.92(5)$ \\
\hline $\mathrm{Si} 2-\mathrm{Si} 1-\mathrm{Nd} 1$ & $116.71(6)$ \\
\hline $\mathrm{Si} 2-\mathrm{Si} 1-\mathrm{Nd} 1^{\mathrm{xvii}}$ & $119.56(6)$ \\
\hline $\mathrm{Si} 2-\mathrm{Si} 1-\mathrm{Nd} 2^{\mathrm{xiii}}$ & $63.78(5)$ \\
\hline $\mathrm{Si} 2-\mathrm{Si} 1-\mathrm{Nd} 2^{\mathrm{ii}}$ & $135.29(6)$ \\
\hline $\mathrm{Si} 2-\mathrm{Si} 1-\mathrm{Nd} 2^{\mathrm{i}}$ & $65.57(5)$ \\
\hline $\mathrm{Si} 2-\mathrm{Si} 1-\mathrm{Nd}^{\mathrm{vi}}$ & $137.48(7)$ \\
\hline $\mathrm{Si} 2-\mathrm{Si} 1-\mathrm{Nd} 3^{\mathrm{ii}}$ & $63.95(4)$ \\
\hline $\mathrm{Si} 2-\mathrm{Si} 1-\mathrm{Nd} 3^{\mathrm{x}}$ & $69.39(5)$ \\
\hline $\mathrm{Nd} 1^{\mathrm{xii}}-\mathrm{Si} 2-\mathrm{Nd} 3^{\mathrm{x}}$ & $135.36(6)$ \\
\hline $\mathrm{Nd} 2-\mathrm{Si} 2-\mathrm{Nd} 1^{\mathrm{xii}}$ & $70.04(4)$ \\
\hline $\mathrm{Nd} 2^{\mathrm{xii}}-\mathrm{Si} 2-\mathrm{Nd} 1^{\mathrm{xii}}$ & $142.04(5)$ \\
\hline $\mathrm{Nd} 2{ }^{\mathrm{i}}-\mathrm{Si} 2-\mathrm{Nd} 1^{\mathrm{xii}}$ & $68.47(3)$ \\
\hline $\mathrm{Nd} 2-\mathrm{Si} 2-\mathrm{Nd} 2^{\text {xiii }}$ & $133.24(5)$ \\
\hline $\mathrm{Nd} 2-\mathrm{Si} 2-\mathrm{Nd} 2^{\mathrm{i}}$ & $81.83(4)$ \\
\hline $\mathrm{Nd} 2^{\mathrm{xii}}-\mathrm{Si} 2-\mathrm{Nd} 2^{\mathrm{i}}$ & $134.00(5)$ \\
\hline $\mathrm{Nd} 2^{\mathrm{xii}}-\mathrm{Si} 2-\mathrm{Nd} 3^{\mathrm{ii}}$ & 80.09 (4) \\
\hline $\mathrm{Nd} 2^{\mathrm{xii}}-\mathrm{Si} 2-\mathrm{Nd} 3^{\mathrm{x}}$ & $82.54(4)$ \\
\hline $\mathrm{Nd} 2-\mathrm{Si} 2-\mathrm{Nd} 3^{\mathrm{ii}}$ & $139.78(6)$ \\
\hline $\mathrm{Nd} 2^{\mathrm{i}}-\mathrm{Si} 2-\mathrm{Nd} 3^{\mathrm{ii}}$ & $87.23(4)$ \\
\hline $\mathrm{Nd} 2-\mathrm{Si} 2-\mathrm{Nd}^{\mathrm{x}}$ & $78.46(4)$ \\
\hline $\mathrm{Nd} 2^{\mathrm{i}}-\mathrm{Si} 2-\mathrm{Nd}^{\mathrm{x}}$ & $76.59(3)$ \\
\hline $\mathrm{Nd} 22^{\text {xiii }}-\mathrm{Si} 2-\mathrm{Nd} 3$ & $80.12(4)$ \\
\hline $\mathrm{Nd} 2-\mathrm{Si} 2-\mathrm{Nd} 3$ & $85.51(4)$ \\
\hline $\mathrm{Nd} 2-\mathrm{Si} 2-\mathrm{Nd} 3$ & $140.17(6)$ \\
\hline $\mathrm{Nd} 3-\mathrm{Si} 2-\mathrm{Nd} 1{ }^{\mathrm{xii}}$ & $71.70(3)$ \\
\hline $\mathrm{Nd} 3^{\mathrm{ii}}-\mathrm{Si} 2-\mathrm{Nd} 1^{\mathrm{xii}}$ & $69.93(3)$ \\
\hline $\mathrm{Nd} 3-\mathrm{Si} 2-\mathrm{Nd}^{3}$ & $137.09(5)$ \\
\hline $\mathrm{Nd} 3-\mathrm{Si} 2-\mathrm{Nd} 3^{\mathrm{ii}}$ & $78.55(4)$ \\
\hline $\mathrm{Nd} 3^{\mathrm{ii}}-\mathrm{Si} 2-\mathrm{Nd} 3^{\mathrm{x}}$ & $136.04(5)$ \\
\hline $\mathrm{Si} 1-\mathrm{Si} 2-\mathrm{Nd} 1^{\mathrm{xii}}$ & $120.80(6)$ \\
\hline $\mathrm{Si} 1-\mathrm{Si} 2-\mathrm{Nd} 2^{\mathrm{i}}$ & $66.76(5)$ \\
\hline $\mathrm{Si} 1-\mathrm{Si} 2-\mathrm{Nd} 2^{\mathrm{xiii}}$ & $67.28(5)$ \\
\hline $\mathrm{Si} 1-\mathrm{Si} 2-\mathrm{Nd} 2$ & $135.73(6)$ \\
\hline $\mathrm{Si} 1-\mathrm{Si} 2-\mathrm{Nd} 3^{\mathrm{ii}}$ & $71.20(5)$ \\
\hline
\end{tabular}


supporting information

$\begin{array}{llll}\mathrm{Si} 2-\mathrm{Nd} 2-\mathrm{Si}^{\mathrm{x}} & 100.44(2) & \mathrm{Si} 1-\mathrm{Si} 2-\mathrm{Nd} 3 & 138.40(7) \\ \mathrm{Si} 2-\mathrm{Nd} 2-\mathrm{Si}^{2} & 93.45(5) & \mathrm{Si} 1-\mathrm{Si} 2-\mathrm{Nd} 3^{\mathrm{x}} & 64.85(5)\end{array}$

Symmetry codes: (i) $-y+1,-x+1,-z+1 / 2$; (ii) $-y+3 / 2, x+1 / 2, z+1 / 4$; (iii) $x+1 / 2,-y+3 / 2,-z+3 / 4$; (iv) $-x+1,-y+1, z+1 / 2$; (v) $-x+1,-y+2, z+1 / 2$; (vi) $-y+2$, $-x+1,-z+1 / 2$; (vii) $-y+3 / 2, x-1 / 2, z+1 / 4$; (viii) $y, x,-z+1$; (ix) $x-1 / 2,-y+3 / 2,-z+3 / 4$; (x) $-x+3 / 2, y-1 / 2,-z+1 / 4$; (xi) $y, x,-z$; (xii) $y-1 / 2,-x+3 / 2, z-1 / 4$; (xiii) $-x+3 / 2, y+1 / 2,-z+1 / 4$; (xiv) $-y+1,-x+2,-z+1 / 2$; (xv) $-x+1,-y+1, z-1 / 2$; (xvi) $-x+1,-y+2, z-1 / 2$; (xvii) $y+1 / 2,-x+3 / 2, z-1 / 4$. 